\begin{tabular}{|c|c|c|c|}
\hline \multicolumn{4}{|c|}{ REPORT DOCUMENTATION PAGE } \\
\hline \multicolumn{4}{|c|}{ 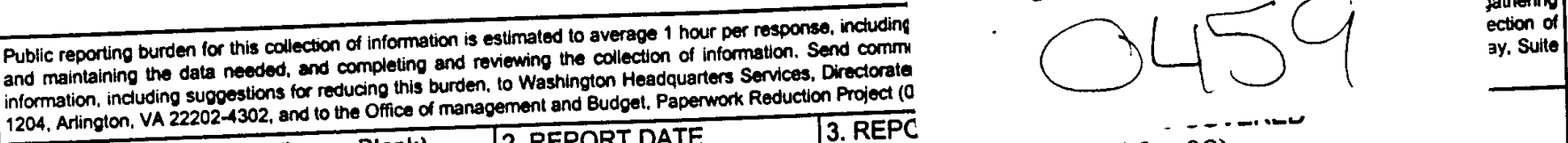 } \\
\hline 1. AGENCY USE ONLY (Leave Blank) & \begin{tabular}{|l|l} 
2. REPORT DATE & 3 \\
11 April 1998
\end{tabular} & $\begin{array}{l}\text { 3. REPC } \\
\text { Final (15 Mar 96- } 14 \text { Mar 98) }\end{array}$ & \\
\hline \multicolumn{3}{|c|}{$\begin{array}{l}\text { 4. TITLE AND SUBTITLE } \\
\text { Computational Methods for the Simulation of Nonconvex Variational Problems } \\
\text { with Applications to Smart Materials }\end{array}$} & $\begin{array}{l}\text { DING NUMBERS } \\
20-96-1-0090\end{array}$ \\
\hline \multicolumn{3}{|l|}{$\begin{array}{l}\text { 6. AUTHORS } \\
\text { Dr. James C. Turner, Jr. }\end{array}$} & \\
\hline \multicolumn{3}{|c|}{$\begin{array}{l}\text { 7. PERFORMING ORGANIZATION NAME(S) AND ADDRESS(ES) } \\
\text { Florida A\&M University } \\
\text { Tallahassee, FL } 32307\end{array}$} & $\begin{array}{l}\text { FORMING ORGANIZATION } \\
\text { ORT NUMBER }\end{array}$ \\
\hline \multicolumn{3}{|c|}{$\begin{array}{l}\text { 9. SPONSORING/MONITORING AGENCY NAME(S) AND ADDRESS(ES) } \\
\text { AFOSRNNM } \\
\text { 110 Duncan Avenue, Room B-115 } \\
\text { Bolling Air Force Base, DC } 20332-8080\end{array}$} & $\begin{array}{l}\text { ONSORING/MONITORING } \\
\text { SENCY REPORT NUMBER }\end{array}$ \\
\hline \multicolumn{4}{|c|}{$\begin{array}{l}\text { Bolling Air Force Base, DC } 20332-8080 \\
\text { 11. SUPPLEMENTARY NOTES }\end{array}$} \\
\hline \multicolumn{3}{|c|}{$\begin{array}{l}\text { 12a. DISTRIBUTION AVAILABILITY STATEMENT } \\
\text { Approved for Public Release }\end{array}$} & ISTRIBUTION CODE \\
\hline \multicolumn{4}{|c|}{$\begin{array}{l}\text { 13. ABSTRACT (Maximum } 200 \text { words) } \\
\text { An interdisciplinary research group in modeling, analysis and simulation was } \\
\text { established at Florida A\&M University (FAMU). This group set as its scientific goal } \\
\text { the development of robust computational algorithms that are well suited for } \\
\text { nonconvex variational principles, and other nonlinear problems that arise in the } \\
\text { study of highly nonlinear materials. The equally important second goal of the group } \\
\text { was to enhance the participation of under-represented minorities in applied } \\
\text { disciplines connected to emerging technologies that are important to the Air Force } \\
\text { office of Scientific Research. Mechanisms used for meeting these goals include the } \\
\text { strengthening of the infrastructure for research at FAMU, strengthening of the } \\
\text { existing relationship between AFOSR and FAMU, and enhancing the existing } \\
\text { relationship between EAMU, Carnegie Mellon University, University of Minnesota, and } \\
\text { North Carolina University. This report describes the background and overall } \\
\text { research goals of this project, and delineates the accomplishments made thus far. }\end{array}$} \\
\hline \multicolumn{4}{|l|}{$\begin{array}{l}\text { 14. SUBJECT TERMS } \\
\text { nonconvex, variational, FAMU }\end{array}$} \\
\hline & & . & 16. PRICE CODE \\
\hline \begin{tabular}{l|l} 
17. SECURITY CLASSIFICATION & 18. \\
OF REPORT & $\mathrm{Un}$ \\
Unclassified &
\end{tabular} & $\begin{array}{l}\text { 18. SECURITY CLASSIFICATION } \\
\text { OF THIS PAGE } \\
\text { Unclassified }\end{array}$ & $\begin{array}{l}\text { 19. SECURITY CLASSIFICATION } \\
\text { OF ABSTRACT } \\
\text { Unclassified }\end{array}$ & $\begin{array}{l}\text { 20. LIMITATION OF ABSTRACT } \\
\text { UL }\end{array}$ \\
\hline
\end{tabular}




\title{
COMPUTATIONAL METHODS FOR THE SIMULATION OF NONCONVEX VARIATIONAL PROBLEMS WITH APPLICATIONS TO SMART MATERIAL
}

\begin{abstract}
An interdisciplinary research group in modeling, analysis and simulation was established at Florida A\&M University (FAMU).This group set as its scientific goal the development of robust computational algorithms that are well suited for nonconvex variational principles, and other nonlinear problems that arise in the study of highly nonlinear materials. The equally important second goal of the group was to enhance the participation of under-represented minorities in applied disciplines connected to emerging technologies that are important to the Air Force Office of Scientific Research. Mechanisms used for meeting these goals include the strengthening of the infrastructure for research at FAMU, strengthening of the existing relationship between AFOSR and FAMU, and enhancing the existing relationship between FAMU, Carnegie Mellon University, University of Minnesota, and North Carolina University. This report describes the background and overall research goals of this project, and delineates the accomplishments made thus far.
\end{abstract}

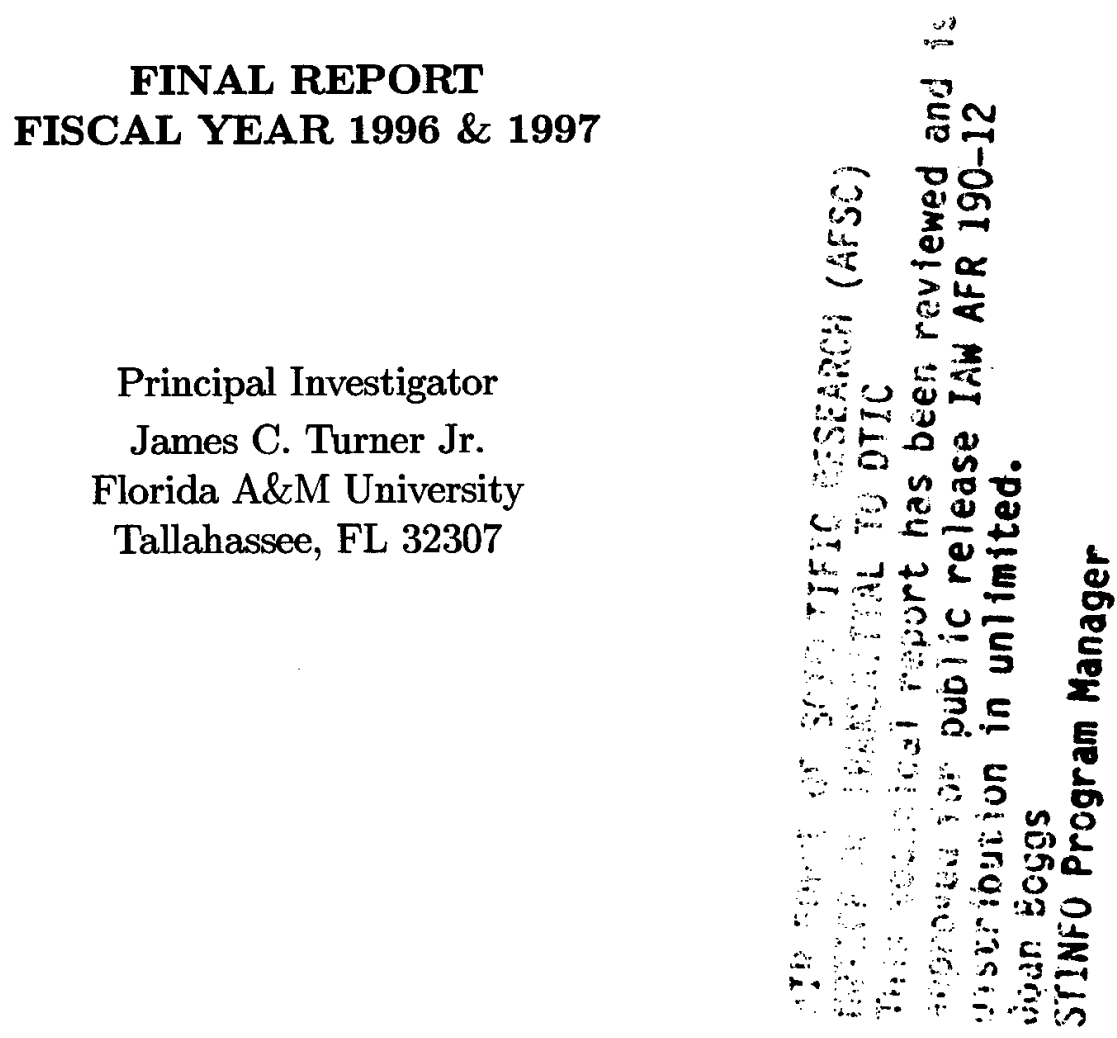




\section{Introduction}

Florida A\&M University has established an interdisciplinary group in modeling, analysis, and simulation. The main scientific goal of this group is the development of robust computational algorithms for nonconvex variational principles, and other nonlinear problems that arise in the study of highly nonlinear materials. This group was composed of James C. Turner Jr. and Yanzhao Cao from the Department of Mathematics at Florida A\&M University, and consultants Richard James (University of Minnesota), David Kinderlehrer (Carnegie Mellon University), Mitchell Luskin (University of Minnesota), and Gregory Forest (University of North Carolina).

An additional goal for Florida A\&M University (FAMU) was the establishment of a Center for Computational Science (CCS). The aim of this Center was to provide world-class education and research in areas that are essential to the nation's scientific progress and economic competitiveness. The mission of the Center is to strive for a productive education and research environment, with innovative educational teaching techniques, effective use of state-of-the-art technology, seminars, visitors, conferences, and publications as its visible signs of success. It was proposed that the CCS become the primary location of activities supported by this grant. Funding from this grant was instrumental in developing an infrastructure for research, where there is

- release time for research faculty;

- summer research activities;

- up-to-date computing facilities;

- visiting scholars';

- support for undergraduate students. 


\section{Scientific Program}

\subsection{Optimization and Control Strategies in Fiber Manufacturing}

The primary objective of this research is to merge novel methods for control of polymer extrusions with focused fiber manufacturing issues. The secondary objective is to develop finite element algorithms that solve specific model equations. When combined with experiments these algorithms yield fundamental relationships between material properties of industrial fluids (agricultural sprays or polymeric liquids) and measurable flow length scales found in laboratory experiments. Each of these objectives involves the development and application of new mathematical and numerical methods for problems defined by the industrial and manufacturing community.

In ploymer extrusion processes, one is often interested in maintaining a quasiuniform temperature, so as to reduce material (extrusion product) inhomogeneity, throughout the extrudate cooling process. As a preliminary step we have studied the following simplified case: seek a uniform temperature distribution at the exit under a steady state condition. To achieve a uniform temperature distribution at the exit we adjusted the heat flux on the surface of the pipe near the exit.

The extrudate in question is assumed to be a viscoelastic fluid of the Oldroyd type, e.g., polymer melts with a fast relaxation mode. Here $\mathbf{u}$ denotes the velocity field, $p$ the pressure field, $T$ the temperature field, and $\tau$ the purely elastic part of the extra stress. Also, $\mathbf{D}=\frac{1}{2}\left(\nabla \mathbf{u}-\nabla \mathbf{u}^{T}\right), \mathbf{W}=\frac{1}{2}\left(\nabla \mathbf{u}+\nabla \mathbf{u}^{T}\right)$ and $D_{a} \tau=$ $(\mathbf{u} \cdot \nabla) \tau+\tau \mathbf{W}-\mathbf{W} \boldsymbol{\tau}-a(\mathbf{D} \tau+\tau \mathbf{D})$, where $-1 \leq a \leq 1$. Next, let $R e$, We and $\omega$ be the Reynolds number, Weissenberg number and retardation parameter, respectively. With these definitions the governing equations for an Oldroyd type fluid, in dimensionless form, is given by the Navier-Stokes equations

$$
\operatorname{Re}(\mathbf{u} \cdot \nabla) \mathbf{u}+\nabla p=(1-\omega) \Delta \mathbf{u}+\operatorname{div} \boldsymbol{\tau}+\mathbf{f}, \quad \text { in } \Omega,
$$

the incompressibility constraint

$$
\operatorname{div} \mathbf{u}=0 \quad \text { in } \Omega
$$

the constitutive equation (Oldroyd Model)

$$
\boldsymbol{\tau}+W e D_{a} \boldsymbol{\tau}=2 \omega \mathbf{D} \text { in } \Omega
$$

the boundary condition (for this preliminary study)

$$
\mathbf{u}=\mathbf{h} \text { on } \Gamma
$$


the energy equation

$$
-\kappa \nabla T+(\mathbf{u} \cdot \nabla) T=\mathbf{Q}+2 \mu\left(\nabla \mathbf{u}+\nabla \mathbf{u}^{T}\right):\left(\nabla \mathbf{u}+\nabla \mathbf{u}^{T}\right) \quad \text { in } \Omega,
$$

and with additional boundary conditions

$$
\begin{aligned}
T & =0 & \text { on } \Gamma_{D}, \\
\frac{\partial T}{\partial \mathbf{n}} & =\mathbf{H}_{N} & \text { on } \Gamma_{N} \cup \Gamma_{0}, \\
\frac{\partial T}{\partial \mathbf{n}} & =\mathbf{g} & \text { on } \Gamma_{C} .
\end{aligned}
$$

In this setting the data functions $\mathbf{f}, \mathbf{Q}, \mathbf{H}_{N}$, and $\mathbf{h}$ are assumed known; the control $\mathbf{g}$ is to be determined so that hot spots are avoided. The constants $\kappa$ and $\mu$ depend on the thermal conductivity coefficient, density, specific heat at constant volume, and viscosity coefficient of the fluid. We assumed that buoyancy effects can be neglected, and thus the temperature variable does not appear in (2.1).

Two means of obtaining a uniform temperature distribution have been considered. The first involves making the gradient of the temperature along the boundary $\Gamma_{0}$ small. Thus, for example, given a velocity field $\mathbf{u}$, we would seek a temperature field $T$ and control field $\mathbf{g}$ such that the functional

$$
M(T, \mathbf{g})=\frac{\alpha}{2} \int_{\Gamma_{0}}\left|\nabla_{s} T\right|^{2} d \bar{\Gamma}+\frac{\kappa \delta}{2} \int_{\Gamma_{C}}|\mathbf{g}|^{2} d \Gamma
$$

is minimized, of course, subject to the constraints imposed by the flow equations $(2.1)-(2.5)$. Here, $\nabla_{s}$ denotes the surface gradient operator, e.g., in $\Re^{2}$, the tangential derivative operator $\partial / \partial_{r}$. The non-negative parameters $\alpha$ and $\delta$ can be used to change the relative importance of the two terms appearing in the definition of $M$. These constants may also be used as penalty parameters. The appearance of the control $\mathbf{g}$ in the definition of $M$ is necessary because we are not imposing a priori limits on the size of this control. The minimization of (2.9) results in a quasi-uniform temperature distribution along the boundary segment $\Gamma_{0}$. This is a result of the fact that surface derivatives of the temperature are forced to be small. Another means of achieving the desired result is to try to directly force the temperature field itself to be quasi-uniform. In this setting, given a velocity field $\mathbf{u}$, we would seek a temperature field $T$ and a control field $\mathbf{g}$ such that the functional

$$
\Im(T, \mathbf{g})=\frac{1}{2 \gamma} \int_{\Gamma_{0}}\left|T-T_{d}\right|^{2} d \Gamma+\frac{\kappa \delta}{2} \int_{\Gamma_{C}}|\mathbf{g}|^{2} d \Gamma
$$


is minimized subject to $(2.1)-(2.8)$, where $T_{d}$ is some desired temperature distribution, e.g., a quantity close to the average temperature along $\Gamma_{C}$ for the uncontrolled system. Once again the non-negative parameters $\gamma$ and $\delta$ can be used to change the relative importance of the two terms appearing in the definition of $\Im$ as well as to act a penalty parameters.

\subsubsection{The Optimization Problem, Existence of Solutions, An Optimal- ity System}

Here we give a precise statement of the optimization problem and results obtained. We first note that (2.1)-(2.4) uncouples from (2.5)-(2.8). We may solve for $(\mathbf{u}, p \boldsymbol{\tau})$ from (2.1)-(2.4) once and for all and then substitute them into (2.5)-(2.8). Thus, the only state variable is $T$, i.e., the temperature field, and the only boundary control variable is $\mathbf{g}$. The state and control variables are constrained to satisfy the system (2.5)-(2.8), which are recasted into the following weak form: find $T \in H_{D}^{1}(\Omega)=\left\{S \in H^{1}(\Omega): S=0\right.$ on $\left.\Gamma_{D}\right\}$ such that

$$
\kappa a(T, S)+c(\mathbf{u}, T, S)=(\mathbf{Q}, S)+\kappa(\mathbf{g}, S)_{\Gamma_{C}}+\kappa\left(\mathbf{H}_{N}, S\right)_{\Gamma_{N}} \quad \forall S \in H_{D}^{1}(\Omega),
$$

where

$$
\mathbf{Q}=\overline{\mathbf{Q}}+2 \mu\left(\nabla \mathbf{u}+(\nabla \mathbf{u})^{T}\right):\left(\nabla \mathbf{u}+(\nabla \mathbf{u})^{T}\right) .
$$

For each possible control function $\mathrm{g}$, we have established the existence of a unique corresponding state function $T$.

Lemma 2.1. For every $\mathbf{g} \in L^{2}\left(\Gamma_{C},\right)$, there exists a unique $T \in H_{D}^{1}(\Omega)$ such that (2.11) is satisfied. Moreover,

$$
\|T\|_{1}+\|T\|_{0, \Gamma_{C}} \leq C\left(\|\mathbf{g}\|_{0, \Gamma_{C}}+\|\mathbf{Q}\|_{0}+\left\|\mathbf{H}_{N}\right\|_{0, \Gamma_{N}}\right) .
$$

The admissibility set is defined by

$$
U_{a d}=\left\{(T, \mathbf{g}) \in H_{0}^{1}(\Omega) \times L^{2}\left(\Gamma_{C}\right): \Im(T, \mathbf{g})<\infty, \quad(2.11) \text { is satisfied }\right\}
$$

Then, $(\hat{T}, \hat{g}) \in U_{a d}$ is call an optimal solution if there exists $\varepsilon>0$ such that

$$
\Im(\hat{T}, \hat{g}) \leq \Im(T, \mathbf{g}) \quad \forall(T, \mathbf{g}) \in U_{a d}
$$


satisfying

$$
\|T-\hat{T}\|_{1}+\|\mathbf{g}-\hat{\mathbf{g}}\|_{0, \Gamma_{C}} \leq \varepsilon
$$

Making use of the Lemma, we obtained existence and uniqueness of optimal solutions. We also obtained the optimality condition

$$
g=-\left.\frac{1}{\delta} \Phi\right|_{\Gamma_{C}}
$$

where $\boldsymbol{\Phi}$ is the solution of the adjoint state equation

$$
\kappa a(R, \boldsymbol{\Phi})+c(\mathbf{u}, R, \boldsymbol{\Phi})-\frac{1}{\delta}\left(R, T-T_{d}\right)_{\Gamma_{C}}=0 \quad \forall R \in H_{D}^{1}(\Omega) .
$$

Elimination $g$ from (2.11) and combining with (2.13), we obtain the optimality system

$$
\kappa a(T, S)+c(\mathbf{u}, T, S)+\frac{\kappa}{\delta}(\mathbf{\Phi}, S)_{\Gamma_{C}}=(\mathbf{Q}, S)+\kappa\left(\mathbf{H}_{N}, S\right)_{\Gamma_{N}} \quad \forall S \in H_{D}^{1}(\Omega),
$$

and

$$
\kappa a(R, \boldsymbol{\Phi})+c(\mathbf{u}, R, \boldsymbol{\Phi})-\frac{1}{\delta}\left(R, T-T_{d}\right)_{\Gamma_{C}}=0 \quad \forall R \in H_{D}^{1}(\Omega) .
$$

Thus, the optimal state, i.e., the temperature distribution $T$, can be found by solving the coupled system (2.14)-(2.15), which also provides the optimal co-state $\Phi$. The optimal control $\mathbf{g}$ can then be deduced from (2.12).

\subsubsection{Finite Element Approximation, Error Estimates, and Iterative Methods}

Next, we constructed a finite element algorithm for determining approximations of the optimality system (2.14) - (2.15) as follows: seek $T^{h} \in W^{h}$ and $\Phi^{h} \in W^{h}$ such that

$\kappa a\left(T^{h}, S^{h}\right)+c\left(\mathbf{u}, T^{h}, S^{h}\right)+\frac{\kappa}{\delta}\left(\Phi^{h}, S^{h}\right)_{\Gamma_{C}}=\left(\mathbf{Q}, S^{h}\right)+\kappa\left(\mathbf{H}_{N}, S^{h}\right)_{\Gamma_{N}} \quad \forall S^{h} \in W^{h}$

and

$$
\kappa a\left(R^{h}, \Phi^{h}\right)+c\left(\mathbf{u}, R^{h}, \Phi^{h}\right)-\frac{1}{\delta}\left(R^{h}, T^{h}-T_{d}\right)_{\Gamma_{C}}=0 \quad \forall R^{h} \in W^{h} .
$$

Although the optimality system is linear, the coupling of $\mathbf{u}$ and $\boldsymbol{\Phi}$ makes the derivation of the error estimates nontrivial. To obtain our error estimates we applied the Brezzi-Rappaz-Raviart theory. 
Theorem 2.2. Let $(T, \Phi)$ and $\left(T^{h}, \Phi^{h}\right)$ be the solutions of (2.14)-(2.15), and (2.16)-(2.17), respectively. Assume that $T, \boldsymbol{\Phi} \in H^{m+1}(\Omega) \cap H_{D}^{1}(\Omega)$ for some $1 \leq m \leq k$; also that the approximation property

$$
\inf _{S^{h} \in W^{h}}\left\|S-S^{h}\right\|_{1} \leq C h^{m}\|S\|_{m+1} \quad \forall S \in H_{D}^{1}(\Omega) \text { and } 0 \leq m \leq k
$$

holds. Then,

$$
\left\|T-T^{h}\right\|_{1}+\left\|\mathbf{\Phi}-\boldsymbol{\Phi}^{h}\right\|_{1} \leq C \max \left\{\frac{1}{\delta}, \frac{1}{\gamma}, 1\right\} h^{m}\left(\|Q\|_{m-1}+\left\|H_{N}\right\|_{\Gamma_{N}, m-1 / 2}+\left\|T_{d}\right\|_{\Gamma_{N}, m-1 / 2}\right)
$$

where $C$ is independent of $h, \delta, \gamma, T$, and $\mathbf{\Phi}$.

An iterative algorithm for solving (2.14)-(2.15) is defined as follows: choose $\boldsymbol{\Phi}^{(0)}$; for $n=1,2, \ldots$, solve, for $T^{(n)}$, $\kappa a\left(T^{(n)}, S\right)+c\left(\mathbf{u}, T^{(n)}, S\right)=-\frac{\kappa}{\delta}\left(\mathbf{\Phi}^{(n-1)-}, S\right)_{\Gamma_{C}}+(Q, S)+\kappa\left(H_{N}, S\right)_{\Gamma_{N}} \forall S \in H_{D}^{1}(\Omega) ;$ then solve, for $\Phi^{(n)}$,

$$
\kappa a\left(R, \Phi^{(n)}\right)+c\left(\mathbf{u}, R, \Phi^{(n)}\right)=\frac{1}{\delta}\left(R, T^{(n)}-T_{d}\right)_{\Gamma_{C}} \forall R \in H_{D}^{1}(\Omega) .
$$

The convergence of this algorithm was obtained by observing that it is effectively a gradient method for the following minimization problem: find $g \in L^{2}\left(\Gamma_{C}\right)$ such that $\kappa(\mathbf{g}) \equiv \Im(T(\mathbf{g}), \mathbf{g})$ is minimized where $T(\mathbf{g}) \in H_{D}^{1}(\Omega)$ is defined as the solution of (2.11). 


\subsubsection{Computational Techniques}

Computational results have been obtained using the most powerful computational technique developed thus far, and making effective use of high-performance computer technology. In particular, computational results for a model with an Oldroyd type fluid have been obtained, demonstrating the effectiveness of the theory and code as well as their potential applicability to problems of interest to the Air Force Office of Scientific Research.

We end this section by presenting computational examples considered. Let $\Omega$ be the unit square $(0,1) \times(0,1)$. Let $\Gamma=\Gamma_{C} \cup \Gamma_{D} \bigcup \Gamma_{N} \bigcup \Gamma_{O}$ be as shown in figure 1 .

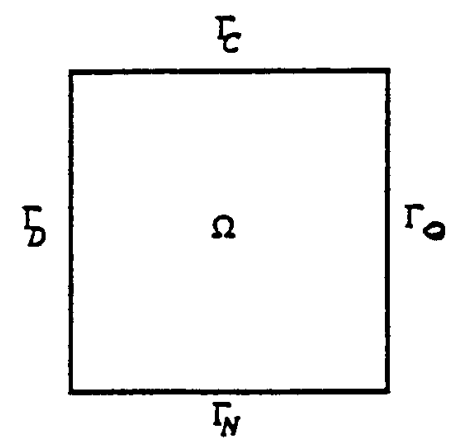

Figure 1. Computational domain 
The finite element spaces $W^{h}$ are chosen to be piecewise linear elements on a triangular mesh. All the numerical results make use of the following parameters and data:

$$
\text { parameters: } \quad \kappa=1 / 0.73 ; \quad \operatorname{Re}=1 ; \quad \omega=1 / 2
$$

$$
\text { boundary data: } \quad T=1 \quad \text { on } \Gamma_{D} ; \quad \frac{\partial T}{\partial n}=0 \quad \text { on } \Gamma_{N} \bigcup \Gamma_{O}
$$

$$
\text { heat source : } \begin{aligned}
Q= & -\frac{5 \varkappa}{2}\left(9 \pi^{2} \cos (3 \pi x) \cos ^{2}(\pi y)-4 \pi^{2} \sin ^{2}\left(\frac{3}{2} \pi x\right) \cos (2 \pi y)\right) \\
& +\left(1-y^{2}\right) \frac{15 \pi}{2} \sin (3 \pi x) \cos ^{2}(\pi y)
\end{aligned}
$$

volcity profile: $\quad \mathbf{u}=\left(1-y^{2}, 0\right)$,

$$
\text { elastic extra stress profile: } \quad \tau=\left(\begin{array}{ll}
0 & -y \\
-y & -4 y^{2}
\end{array}\right)
$$

$$
\text { pressure profile: } \quad p=4\left(1-y^{2}\right)-x \text {. }
$$

In the functional (2.10) we choose

$$
T_{d}=3.5 \text {. }
$$


For the data given above, the exact solution of the uncontrolled problem, i.e., for

$$
\frac{\partial T}{\partial n}=0 \quad \text { on } \Gamma_{C}
$$

is given by $T=5 \sin ^{2}\left(\frac{3}{2} \pi x\right) \cos ^{2}(\pi y)+1$.

We compare the temperature distribution in the uncontrolled case with the optimal temperature distribution in the controlled case for which

$$
\frac{\partial T}{\partial n}=g \quad \text { on } \Gamma_{C}
$$

where $g$ is the control such that the functional (2.10) is minimized. Approximations to the optimal state and co-state are computed form (2.14)-(2.15); the approximate optimal control $g^{h}$ is then obtained from $(2.12)$, i.e., $g^{h}=-\left.(1 / \delta) \Phi\right|_{\Gamma_{c}}$. All of the computational results shown below were obtained with the use of mesh size $h=\frac{1}{19}$. Of course, calculations with varying mesh sizes were performed. Since these merely verified the errors estimates, we do not report on them here.

Specifically, figures 2-4 deal with the following cases:

2. Exact, uncontrolled temperature contours and; Optimal temperature;
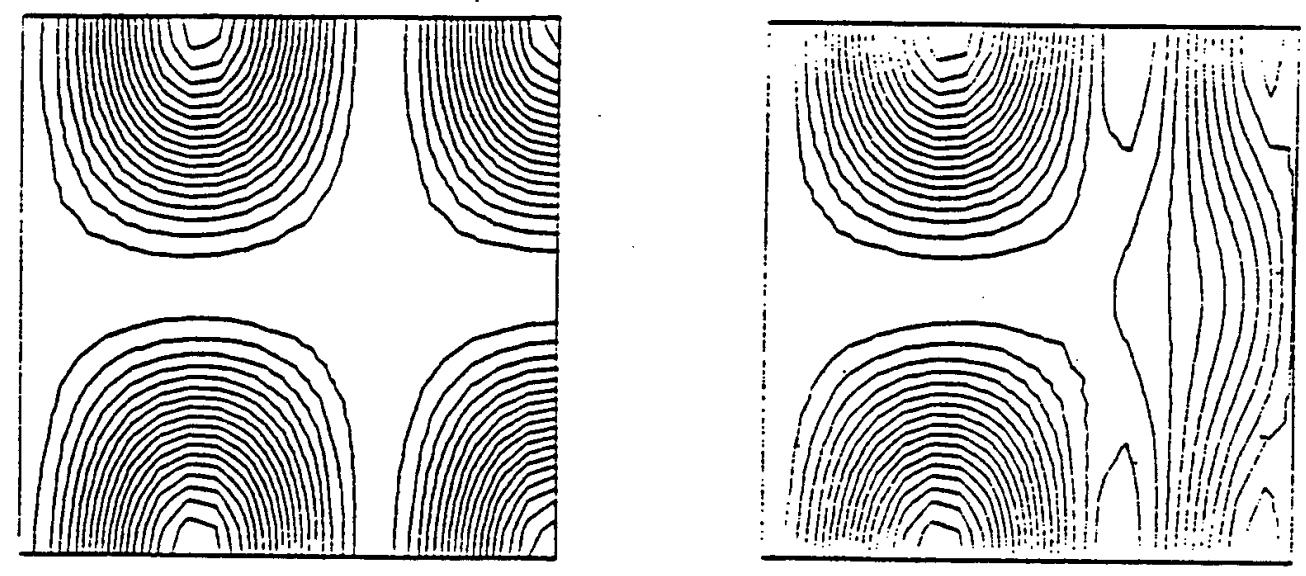

Figure 2. Temperature contours: uncontroled and controled.

Optimal boundary control on $\Gamma_{C}$.

( $\Gamma_{C}$ is the top boundary segment.)

( $\Gamma_{O}$ is the right boundary segment.) 
3. Exact, uncontrolled temperature surfaces and; Optimal temperature;
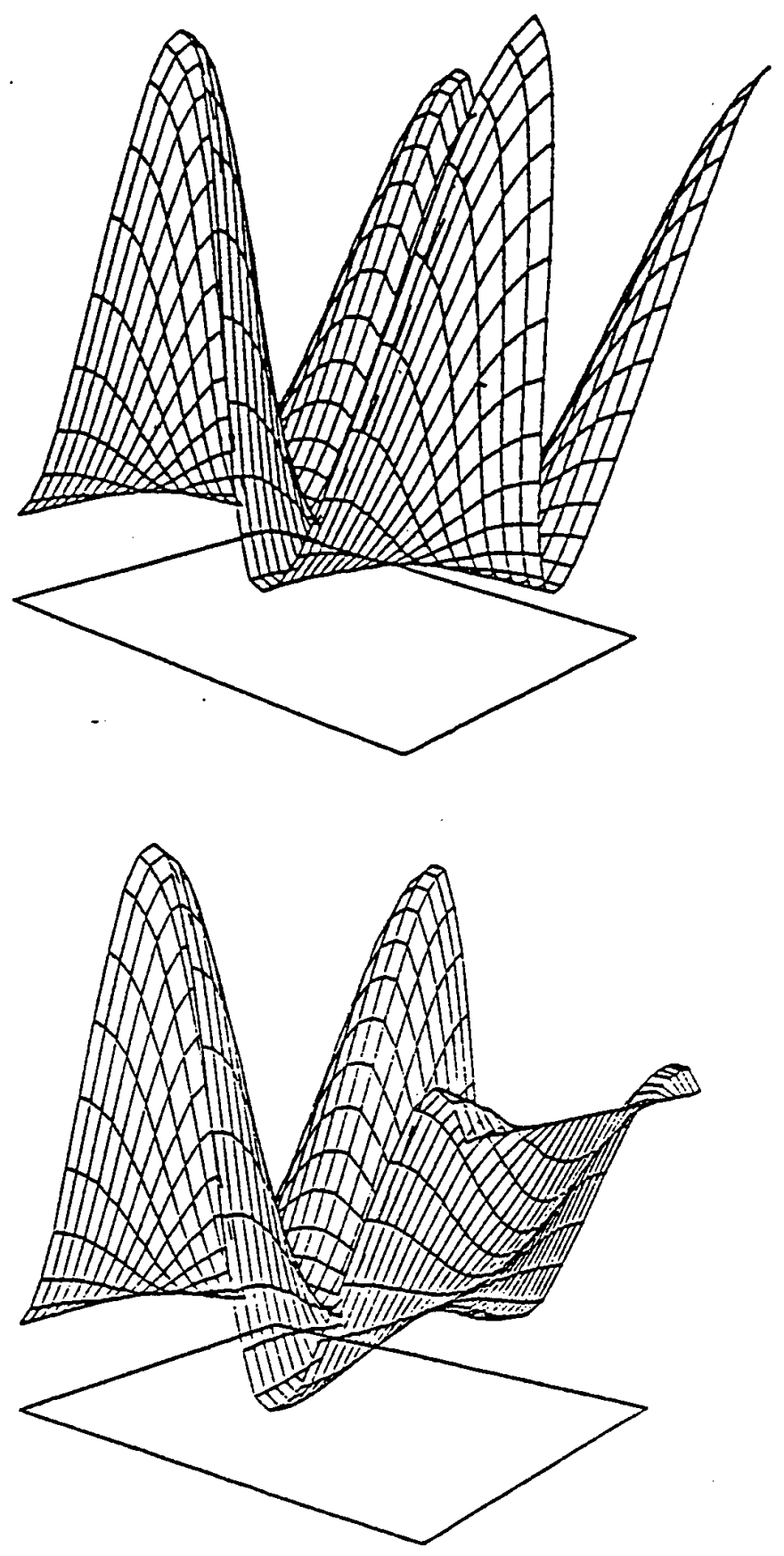
4. Optimal boundary control.

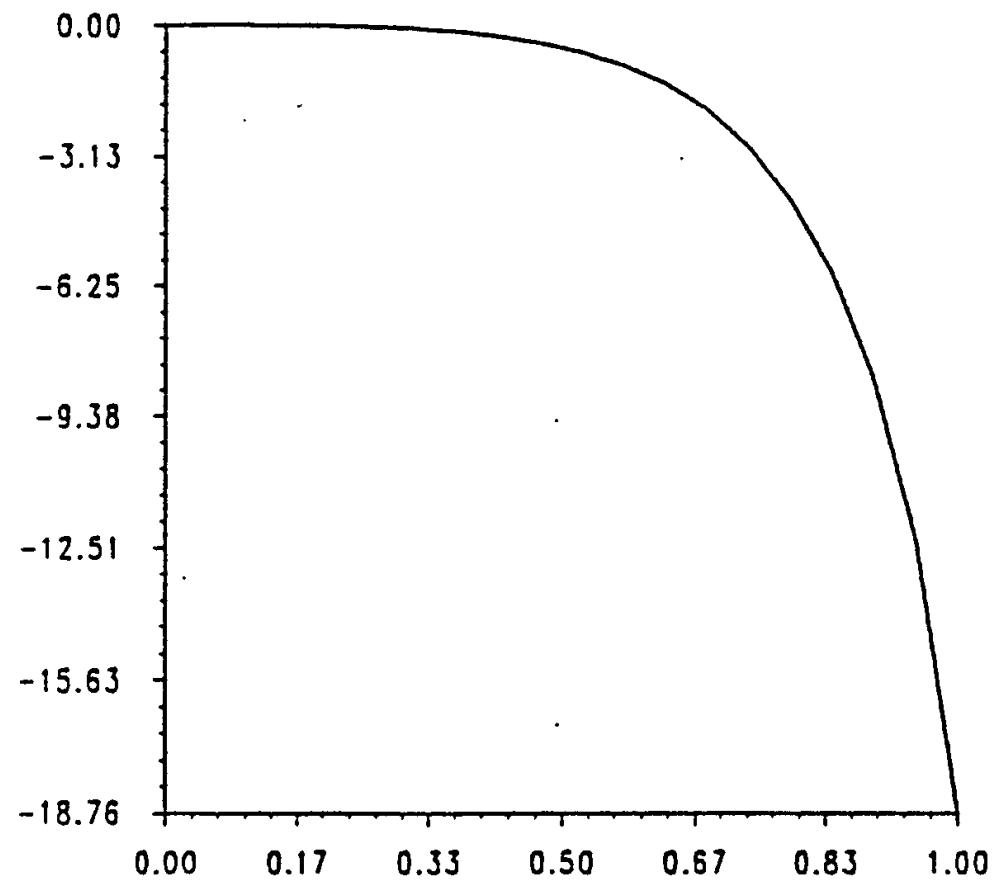

Figure 4. Optimal boundary control on $\Gamma_{C}$. 


\subsection{Simulation of Magnetoelastic Interactions}

Terfenol-D, which is $\operatorname{Tb}_{x} D y_{1-x} F e_{2}, x \cong 0.3$, is a magnetoelastic material exhibiting a complicated microstructure that responds easily and dramatically to an applied magnetic field. It has the largest known magnetoelastic coupling, with huge magetostriction and small magnetomechanical losses, giving it extraordinary promise for use as an actuator and sensor material. Its microstructure or fine scale morphology has a clear role in its macroscopic behavior. Two issues are paramount. First, the presence of several stable variants at a given temperature reflects a complicated potential well structure for the free energy of the material. Second, the presence of spatially oscillatory behavior at the small scale suggests competition between the free energy of the material and loading or other environmental effects. Both of these features represent highly nonlinear processes.

For large specimens, such as those encountered in usual actuator applications, the presence of complicated domain structures has hindered the usefulness of theories which seek to incorporate elastic effects. A theory of magnetostriction which is micromagnetic in nature has been developed by consultants Richard James and David Kinderlehrer. The focus is on the precise specification of the potential wells of the anisotropy energy. There are many parallels between shapememory and giant magnetostrictive behavior. A key difference is that shape memory materials undergo a first order transition resulting in 2-15\% strain while Terfenol suffers a second order transition with only .25\% strain.

By advancing and implementing analytical methods it has been shown that the theory of magnetostriction successfully predicts the equilibrium microstructures observed in Terfenol-D. The theory furthermore suggests a mechanism for the giant magnetostriction related to exchange of stability of the variants within a laminate or among laminate systems on which a magnetic field has been applied.

To understand this phenomenon more completely, in particular away from equilibrium, the interdisciplinary group has established a broad program composed of experiment, theory, and simulation.

\subsubsection{Simulation Program}

The energy of the magnetoelastic systems is described in terms of a free energy of the form

$$
E(y, \mathbf{m}, \mathbf{H})=\int_{\Omega}\{W(\nabla y(x), \mathbf{m}(y(x), x)-\mathbf{H} \cdot \mathbf{m}(y)) \operatorname{det} \nabla y(x)\} d x+\int_{\Re^{3}}|\nabla u|^{2} d x
$$


$|\operatorname{det} \nabla y(x) \mathbf{m}|=1$ in $\Omega$ and $\Delta u=\operatorname{div}\left(\mathbf{m} \chi_{y(\Omega)}\right)$ in $\Re^{3}$,

where $y(x)$ is the elastic deformation, $\Omega \subset \Re^{3}$ is a reference domain, $\mathbf{m}(y)$ the magnetization, and $\mathbf{H}$ the applied magnetic field. The second equation in (2.20) represents the magnetostatic equations from Maxwell's Theory. Since we are dealing with a large body approximation, exchange energy is ignored. The stored energy density $W(A, \mathbf{m}, x)$ is highly nonconvex and gives rise to a functional which is not lower semicontinuous.

The simulation program started with the simulation of hysteretic behavior in two dimensions for

$$
\begin{aligned}
E(\mathbf{H}, \mathbf{m}) & =\int_{\Omega}(\phi(\mathbf{m}, x)-\mathbf{H} \cdot \mathbf{m}) d x+\frac{1}{2} \int_{\Re^{3}}|\nabla u|^{2} d x \\
\Delta u & =\operatorname{div} \mathbf{m} \chi_{\Omega} \quad \text { in } \Re^{2} \quad|\mathbf{m}|=1 \quad \text { in } \Omega .
\end{aligned}
$$

where an applied field is introduced in the system and cycled through a range of values.

Using methods of nonlinear analysis computational results and diagnostics have been explored. Since the energy picture is mesh independent, computing on a fairly coarse grid suffices to establish its character. Kinderlehrer and Ma determined analytically the hysteresis loop. For a magnetic system, this analysis rests on the introduction of a shadow energy for a simplified version of the system. It is believed that this simplified version may suggest possible dispersive interactions which may be attributed to a shape-memory or pseudoelastic body. It was observed that the simulation displays a modified Stoner-Wohlfarth scenario. This means that the system evolves through stable/metastable/unstable/stable regimes. By modeling the nature of the instability one is able to determine the critical field event.

These collaborators than modified the simulation to include magnetoelastic effects, in the context of linear elasticity. The linear elastic magnetostrictive energy has the form

$$
\begin{aligned}
\phi(\varepsilon, \alpha) & =\phi_{\text {elastic }}(\varepsilon)+\phi_{\text {em }}(\varepsilon, \alpha)+\phi_{a n}(\alpha) \\
\varepsilon & =\frac{1}{2}\left(\nabla u+\nabla u^{T}\right), \quad m=M \alpha, \quad|\alpha|=1,
\end{aligned}
$$

where 


$$
\begin{aligned}
\phi_{e l a s t i c}(\varepsilon) & =\frac{1}{2} \sum \varepsilon_{i i}^{2}+c_{12}\left(\varepsilon_{11} \varepsilon_{22}+\varepsilon_{22} \varepsilon_{33}+\varepsilon_{33} \varepsilon_{11}\right)+\frac{1}{2} c_{44}\left(\varepsilon_{12}^{2}+\varepsilon_{23}^{2}+\varepsilon_{13}^{2}\right), \\
\phi_{e m}(\varepsilon, \alpha) & =b_{0} \operatorname{tr} \varepsilon+b_{1} \sum \varepsilon_{i i} \alpha_{i}^{2}+b_{2}\left(\varepsilon_{11} \alpha_{1} \alpha_{2}+\varepsilon_{23} \alpha_{2} \alpha_{3}+\varepsilon_{13} \alpha_{1} \alpha_{3}\right), \\
\phi_{a n}(\alpha) & =K \sum \alpha_{i}^{4} .
\end{aligned}
$$

A two dimensional model was obtained by projecting onto the $(0-11)$ plane, making the assumption that $\phi_{\text {elastic }}$ is isotropic, and changing coordinates so that $(-211)$ is $x_{1}$ and (111) is $x_{2}$. In this setting

$$
\phi^{+}(\varepsilon, \alpha)=\phi_{\text {elastic }}(\varepsilon)+\phi_{e m}(\varepsilon, \alpha)+\phi_{a n}(\alpha)
$$

with

$$
\begin{aligned}
\phi_{\text {elastic }}(\varepsilon) & =\mu \sum \varepsilon_{i j}^{2}+\frac{1}{2} \lambda(\operatorname{tr} \varepsilon)^{2}, \mu>0, \lambda>0, \\
\phi_{e m}^{+}(\varepsilon, \alpha) & =b\left(\varepsilon_{11}-\gamma \varepsilon_{12}\right)\left(\left(\alpha_{1}\right)^{2}-\gamma \alpha_{1} \alpha_{2}\right)-b^{\prime} \sum \varepsilon_{i j} \alpha_{i} \alpha_{j}, \\
\phi_{a n}^{+}(\alpha) & =\kappa\left(\left(\alpha_{1}\right)^{2}-\gamma \alpha_{1} \alpha_{2}\right)^{2}, \kappa>0 .
\end{aligned}
$$

Here $\gamma=2 \sqrt{2}, b<0, b^{\prime}>0$, with $b$ approximately equal to $-b_{2}$, and $b^{\prime}$ is roughly $b_{1}-b_{2}$. To calculate the magnetostriction in the original $(-211)$ direction, now the $(1,0)$ direction, one must first compute the average of $\varepsilon$ and then apply the formula. Thus, one has

$$
\sum \varepsilon_{i j} \beta_{i} \beta_{j}=\bar{\varepsilon}_{11}
$$

Unfortunately, the above simulations do not give satisfying results, i.e., the energies described above do a poor job of resolving the microstructure. The challenge facing the FAMU group was to develop a better simulation.

\subsubsection{Computational Techniques and Code Development}

The global infimum of the energies described in section 2 are not attained. However, it is assumed that the underlying physical microstructure can be represented by suitable minimizing sequences. In order to develop a computer simulation which can provide us with an efficient and effective selection principle for such 
minimizing sequences, our strategy is to employ the most powerful computational techniques developed thus far, develop new ideas and make effective use of highperformance computer technology. Thus, we have simultaneously been working on two different approaches: (i) a global minimization method based on the idea of simulated annealing; and (ii) a study of the minimization of a relaxed problem where generalized solutions in terms of Young measures for the original nonconvex problem are approximated. Both of these approaches must somehow address the following challenging problem: loss of uniqueness of global minimum, the emergence of abundant local minimum or even saddle points.

A state-of-the-art computer model, developed by Carnegie Mellon researchers, that can simulate unconstrained minimization of the angular variable and the displacement for linear magnetostriction has been developed. The hysteresis diagram is computed using a continuation of solutions with respect to increasing and decreasing the applied magnetic field along the $x_{1}$-axis. Work is currently under way to extend this code to nonlinear magnetostriction.

Educational activities in this area of research included the training of a large number of minority undergraduate and graduate students. We have also offered a special lecture series on modeling and simulation of problems arising in the study of materials science. Also, special discussion groups were held. These activities brought some of the researchers and their research into the classroom.

\subsection{Controllability for Parabolic Equations}

In many problems of interest to materials science and engineering there arises the need of controllability for parabolic equations. The purpose of this research was to investigate exact controllability problems for systems modeled by linear parabolic differential equations. Our aim of investigation has been to examine the limit behavior of the corresponding optimal control problem. Using this technique we obtained a new condition on the exact controllability of the system.

We now give a statement of the problem. Let $\Omega$ be a bounded domain in $R^{d}$. For fixed $T$, let $Q=[0, T] \times \Omega$ and $\Sigma=(0, T) \times \partial \Omega$. Let $A$ be a second order elliptic differential operator defined by

$$
A(y)=-\sum_{i, j=1}^{d} \frac{\partial}{\partial x}\left(a_{i, j}(\mathbf{x}) \frac{\partial y}{\partial x_{j}}(t, \mathbf{x})\right)+c(\mathbf{x}) y
$$

We assume that $c>0$ on $\Omega$ and that the matrix $\left(a_{i, j}(\mathbf{x})\right)$ is symmetric and positive definite. 
With these definitions, we have studied the following initial boundary value problem

$$
\begin{array}{rlr}
\frac{\partial y}{\partial t}+A(y) & =0 \quad \text { in } Q, \\
\frac{\partial y}{\partial \nu} & =u(t, \mathbf{x}) \quad \text { on } \Sigma \\
(y(\mathbf{x}, 0) & =y_{0}(\mathbf{x}) \quad \text { in } \Omega,
\end{array}
$$

where

$$
\frac{\partial}{\partial \nu}=\sum_{i, j=1}^{d} a_{i, j} n_{i} \frac{\partial}{\partial x_{j}}
$$

with $n_{i}$ being the $i^{\text {th }}$ component of the outward unit normal to $\partial \Omega$. We say that the system is exactly controllable for a given state $\hat{y}$ if there exists $u \in L^{2}(\Sigma)$ such that the solution $y$ of problem (2.23) also satisfies

$$
y(T, \cdot)=\hat{y}(\cdot)
$$

Next, we define the cost functional $J_{\varepsilon}$ by

$$
J_{\varepsilon}(u)=\frac{1}{2} \int_{\Omega}(y(T, \mathbf{x}, u)-\hat{y}(\mathbf{x}))^{2} d \mathbf{x}+\frac{\varepsilon}{2} \int_{\Sigma} u^{2}(t, \mathbf{x}) d \mathbf{x} d t
$$

where $y=y(t, x ; u)$ satisfies (2.24). $u_{\varepsilon}(t, x)$ is said to be an optimal control if it satisfies

$$
J_{\varepsilon}\left(u_{\varepsilon}\right)=\inf _{u \in L^{2}(Q)} J_{\varepsilon}(u)
$$

Our weak formulation of problem (2.24) takes the form

$$
\begin{aligned}
\left(\frac{\partial y}{\partial t}, \phi\right)+a(y, \phi) & =\langle u, \phi\rangle \quad \text { for } \phi \in H^{1}(\Omega) \\
y(0, \mathbf{x}) & =y_{0}(\mathbf{x}),
\end{aligned}
$$

where the bilinear form $a: H^{1}(\Omega) \times H^{1}(\Omega) \longrightarrow R$ is given by

$$
a(\phi, \psi)=\int_{\Omega}\left\{\sum_{i, j=1}^{d} a_{i, j}(\mathbf{x}) \frac{\partial \phi}{\partial x_{i}} \frac{\partial \psi}{\partial x_{j}}+c(\mathbf{x}) \phi \psi\right\} d \mathbf{x}
$$


where $A, B, U$, and $V$ are the appropriately chosen admissible sets.

Remark 1. State equations (2.26) and (2.27) are given in an abstract form that may represent partial differential equations or more general functional relations. However, we shall always take them to represent partial differential equations. These equations are also written in strong form. In order to place them in an abstract weak formulation, let $\mathbf{W}$ denote a normed space of test functions $\mathbf{w}(\mathbf{x})=\left(w_{1}, w_{2}\right)$, where

$$
w_{1} \in\left(L_{q}(\Omega)\right)^{r}, \quad w_{2} \in\left(L_{q}(\Omega)\right)^{r^{\prime}} \quad \text { and } \quad \frac{1}{p}+\frac{1}{q}=1
$$

with $1 \leq p \leq \infty$, and $1 \leq q \leq \infty$. We define the operator $h(\mathbf{w}, u, v): \mathrm{W} \rightarrow \Re$ by

$$
h(\mathbf{w}) \equiv \int_{\Omega} f_{0}(w, u(x), M(x, u)) w_{1} d x+\int_{\Gamma} g_{0}(x,(K(\xi, v))(x), v(x)) w_{2} d \mu
$$

where $f_{0} w_{1}$ and $g_{0} w_{2}$ denote inner products in $\Re^{r}$ and $\Re^{\prime}$, respectively. Instead of state equations (2.26) and (2.27) we may consider a single state equation in the weak form

$$
l(\mathbf{w})=h(\mathbf{w}),
$$

where the operator $l$ replaces both operators $L$ and $I$.

Thus, when considering control strategies, we are interested in the problem of minimizing functionals of the form

$$
\Im(w, u, v)=\int_{\Omega} f(w, u(x), M(x, u)) d x+\int_{\Gamma} g(x,(K(\xi, v))(x), v(x)) d \mu
$$

with state equation (in weak form)

$$
l(\mathbf{w})=h(\mathbf{w}) \quad \text { for all } \mathbf{w} \in \mathbf{W},
$$

and constraints

$$
\begin{array}{llll}
M(x, u) \in A, & u(x) \in U & \text { a.e. in } \Omega \\
K(x, u) \in B, & u(x) \in V & \mu-\text { a.e. on } \partial \Omega
\end{array}
$$


Remark 2. Of particular interest is the case where the element $w$ of an admissible triple $(\mathrm{w}, u, v)$ uniquely determines the controls $u$ and $v$.

Remark 3. In practice one may define the control set $U$ by

$$
U \equiv\left\{u \in L_{+}^{\infty}[0, T]:\|u\|_{\infty} \leq M\right\}
$$

for $M>0$.

Control Strategy 1. A special case of the above abstract optimal control problems is: How do we choose a control function $g$, so that a crystal interface will approximate a prescribed desired profile?

Let $\Omega$ be a region, occupied by the crystal. Then, a mathematical description of this control problem proceeds as follows. If $C(x, t)$ is a positive continuous function, and $\psi(t)$ is a continuous positive function for $0 \leq t<T$. Then, a possible cost functional may be one of the form

$$
\Im(\mathbf{u}, \mathbf{g})=J(\mathbf{u})+\frac{1}{2 \delta} \int_{\Gamma_{c w}}\left|\nabla_{s} g\right|^{2} d \Gamma,
$$

in which some desired optimization objective $J(\mathbf{u})$ is considered in conjunction with the cost of applying the required control $g$, i.e., the norm of $g$. Here $\nabla_{s}$ is the surface gradient operator on the interface $\Gamma$ and $\delta$ is a fixed, sufficiently small parameter. The desired optimization objective $J(\mathbf{u})$ that one could consider is the tracking functional for the interface. This functional, with its domain being the interface, has the form

$$
J(\mathbf{u})=A \int_{\Omega} \psi(t)[H(r, t)-C(r, t)]^{2} d \Omega .
$$

Where $A$ is a suitable positive constant, which reflects the different needs of each control strategy.

We wish to minimize $\Im(\mathbf{u})$ subject to some appropriate state equations and boundary conditions.

\section{Control Strategy 2.}

In free-boundary problems one must use adaptive meshing that tracks the interface motion. The difficulty is that these schemes may not be able to handle topological changes without user intervention. This in turn makes real-time control inconvenient during the simulation. Berg, Yezzi, and Tannenbaum have explored an alternative approach [10]. In their approach one begins by writing 
down the equations of motion of the evolving interface directly. In two dimensions, these are the equations of curve evolution. In this setting the moving interface is described by a family of parameterized curves, $C:[0,1] \times\left[0, t_{f}\right] \rightarrow \Re^{2}$. The evolution of the curve describing the interface is governed by

$$
\frac{\partial C}{\partial t}=\alpha(s, t) T+\beta(s, t) N
$$

where $s$ parametrizes the curve, $N$ is the normal vector, $T$ is the tangent vector, and $\alpha$ and $\beta$ are velocity functions. Currently we are only considering the shape of the interface, thus we may take $\alpha=0$. Note that changing $\alpha$ only changes the curve's parametrization, and not its shape. In addition, if we assume that the motion of the curve is determined solely by the local geometry of the curve, then

$$
\frac{\partial C}{\partial t}=\beta(\kappa) N
$$

where $\kappa(s, t)$ is the curvature.

There are several difficulties that one may encounter when seeking numerical solutions of curve evolution problems. For example, if curvature terms are absent or small, the solution will typically develop corners or shocks, even if the initial data is smooth. Osher and Sethian [11-13], and their coworkers, have developed a series of algorithms that successfully address many of the problems encountered in curve evolution problems. These algorithms employ a level set representation of the interface, and are the foundation of the numerical approach developed by Berg, Yessi, and Tannenbaum [14].

The idea of applying the theory of curve evolution for modeling crystal evolution has been considered by Srinivasan, et al. [15]. Similar real-time interface estimation and control techniques have been developed for reactive etching processes by a number of authors. For example, Shaqfeh and Jurgensen [16], and Singh, et al. [17] have done pioneering work in this area. Adalsteinsson and Sethian build and greatly extended these techniques to a novel approach for modeling etching, deposition, and lithography fabrication processes [ 13,18].

It is our intension to explore the possible use of these algorithms for tracking the evolution of the crystal interfaces. The focus of our study has been the development of techniques that directly control the evolving features of the crystal interfaces. This is a challenging task, however the potential benefits are large. The level set techniques show potential for real-time control. 


\subsection{Controllability for Parabolic Equations}

In many problems of interest to materials science and engineering there arises the need of controllability for parabolic equations. The purpose of this research was to investigate exact controllability problems for systems modeled by linear parabolic differential equations. Our aim of investigation has been to examine the limit behavior of the corresponding optimal control problem. Using this technique we obtained a new condition on the exact controllability of the system.

We now give a statement of the problem. Let $\Omega$ be a bounded domain in $R^{d}$. For fixed $T$, let $Q=[0, T] \times \Omega$ and $\Sigma=(0, T) \times \partial \Omega$. Let $A$ be a second order elliptic differential operator defined by

$$
A(y)=-\sum_{i, j=1}^{d} \frac{\partial}{\partial x}\left(a_{i, j}(\mathbf{x}) \frac{\partial y}{\partial x_{j}}(t, \mathbf{x})\right)+c(\mathbf{x}) y .
$$

We assume that $c>0$ on $\Omega$ and that the matrix $\left(a_{i, j}(\mathbf{x})\right)$ is symmetric and positive definite.

With these definitions, we have studied the following initial boundary value problem

$$
\begin{array}{rlr}
\frac{\partial y}{\partial t}+A(y) & =0 \quad \text { in } Q \\
\frac{\partial y}{\partial \nu} & =u(t, \mathbf{x}) \quad \text { on } \Sigma \\
(y(\mathbf{x}, 0) & =y_{0}(\mathbf{x}) \quad \text { in } \Omega,
\end{array}
$$

where

$$
\frac{\partial}{\partial \nu}=\sum_{i, j=1}^{d} a_{i, j} n_{i} \frac{\partial}{\partial x_{j}}
$$

with $n_{i}$ being the $i^{\text {th }}$ component of the outward unit normal to $\partial \Omega$. We say that the system is exactly controllable for a given state $\hat{y}$ if there exists $u \in L^{2}(\Sigma)$ such that the solution $y$ of problem (2.39) also satisfies

$$
y(T, \cdot)=\hat{y}(\cdot) .
$$

Next, we define the cost functional $J_{\varepsilon}$ by 


$$
J_{\varepsilon}(u)=\frac{1}{2} \int_{\Omega}(y(T, \mathbf{x}, u)-\hat{y}(\mathbf{x}))^{2} d \mathbf{x}+\frac{\varepsilon}{2} \int_{\Sigma} u^{2}(t, \mathbf{x}) d \mathbf{x} d t
$$

where $y=y(t, x ; u)$ satisfies $(2.40) . u_{\varepsilon}(t, x)$ is said to be an optimal control if it satisfies

$$
J_{\varepsilon}\left(u_{\varepsilon}\right)=\inf _{u \in L^{2}(Q)} J_{\varepsilon}(u)
$$

Our weak formulation of problem (2.40) takes the form

$$
\begin{aligned}
\left(\frac{\partial y}{\partial t}, \phi\right)+a(y, \phi) & =\langle u, \phi\rangle \quad \text { for } \phi \in H^{1}(\Omega) \\
y(0, \mathbf{x}) & =y_{0}(\mathbf{x}),
\end{aligned}
$$

where the bilinear form $a: H^{1}(\Omega) \times H^{1}(\Omega) \longrightarrow R$ is given by

$$
a(\phi, \psi)=\int_{\Omega}\left\{\sum_{i, j=1}^{d} a_{i, j}(\mathbf{x}) \frac{\partial \phi}{\partial x_{i}} \frac{\partial \psi}{\partial x_{j}}+c(\mathbf{x}) \phi \psi\right\} d \mathbf{x} .
$$

Our main results of this research has been obtained by using an operator representation of the terminal state. That is, we define an operator $R: L^{2}(\Omega) \rightarrow$ $L^{2}(\Omega)$ by

$$
(\varepsilon+R)(y(T)-\hat{y})=\varepsilon(\hat{y}-E(T) v)
$$

where $E(t)$ is the solution operator for the problem

$$
\begin{aligned}
\frac{\partial w}{\partial t}+A(w) & =0 \quad & \text { on } Q & \\
\frac{\partial w}{\partial v} & =0 & & \text { on } \Sigma \\
w(0, \mathbf{x}) & =z(\mathbf{x}) & & \text { in } \Omega
\end{aligned}
$$

i.e. $w(t, \mathbf{x})=E(t) z(\mathbf{x})$.

The following properties of the operator $R$ have been established: $R$ is a compact operator in $L^{2}(\Omega) ; R$ is symmetric and semi-definite; if $\Sigma$ is analytic, then $\operatorname{ker} R=0$. 
Note that system (2.42) - (2.44) is said to be approximately controllable if, for any given $\hat{y}$ and $\delta>0$, there exists a control $u(t, \mathbf{x})$ and a function $y(t, \mathbf{x})$ such that $(y(t, \mathbf{x}), u(t, \mathbf{x}))$ is a solution of $(2.40)$ and

$$
\|y(T, \mathbf{x})-\hat{y}(\mathbf{x})\|<\delta
$$

Approximate controllability is well known for linear parabolic differential equations. However, the proof that we were able to obtain is different from standard proofs, in that it is constructive.

Finally, using properties of $R$ we obtained the following result on exact controllability of system (2.40).

Theorem 2.3. Let $\left(\left\{e_{j}\right\}_{j=1}^{\infty},\left\{\lambda_{j}\right\}_{j=1}^{\infty}\right)$ be the eigensystem of $R$. If

$$
\hat{y}-E(T) y_{0} \in D\left(R^{-1}\right)
$$

then system (2.40) is exactly controllable.

\section{REFERENCES}

\section{References}

[1] M.D. Gunzburger, A.J. Meir, and J.S. Peterson, On the Existence, Uniqueness, and Finite Element Approximation of Solutions of the Equations of Stationary,Incompressible Magnetohydrodynamics, Math. Comp., 1991, (56:523-563)

[2] L. S. Hou, A. J. Meir, Analysis of Some Boundary Optimal Control Problems for the MHD Equations with Mixed Boundary Conditions, Mathematics Department Research Report, 93-01, Simon Fraser University, 1993

[3] A. J. Meir,On the equations of Stationary, Incompressible Magnetohydrodynamics with Mixed Boundary Conditions, Computers Math with Applic, 1993, (25:13-29) 
[4] J. Peterson, On the Finite Element Approximation of Incompressible Flows of an Electrically Conducting Fluid, Numer, Meth. P.D.E.s 4, 1988, 57-68.

[5] N. Winowich and W. Hughes, A Finite Element Analysis of Two Dimensional MHD Flows, Liquid-Metal Flows and magnetohyddrodynamics, H. Branover, P.S. Lykoudis and A. Yakhot (Ed.), AIAA, New York, 1983

[6] M. D. Gunzburger, A. J. Meir, J. Peterson, On the Existence, Uniqueness, and Finite Element Approximation of Solutions of the Equations of Stationary, Incompressible Magnetohydrodynamics, Math. Comp., 1991, 57:123151

[7] R.A. Adams, Sobolev Spaces, Academic Press, New York

[8] V. Girault, R.A. Raviart, Finite Element Methods for Navier-Stokes Equations, Springer-Verlag, Berlin

[9] E. Zeidler, Nonlinear Functional Analysis and its Applications, Vol III, Springer-Verlag, New York

[10] J. Berg, A. Yezzi, A. Tannenbaum, Phase Transitions, Curve Evolution, and the Control of Semiconductor Manufacturing Processes, IMA Preprint Series \#1454, University of Minnesota, February 1997

[11] S. Osher, Riemann Solvers, the Entropy Condition, and Difference Approximations, SIAM J. Numer. Anal. 21, 1984, 217-235

[12] S. J. Osher, J. A. Sethian, Fronts Propagation with Curvature Dependent Speed: Algorithms Based on Hamilton-Jacobi Formulations, J. of Comp. Phys 79, 1988, 12-49

[13] J. A. Sethian, Level Set Methods: Evolving Interfaces in Geometry, Fluid Mechanics, Computer Vision, and Materials Science, Cambridge University Press, 1996

[14] J. Berg, A. Yezzi, A. Tannenbaum, Phase Transitions, Curve Evolution, and the Control of Semiconductor Manufacturing Processes, Proceedings of the 35th IEEE Conference on Decision and Control, 1996, kobe, Japan, 3376-3381 
[15] A. Srinivasan, C. Batur, B. N. Rosenthal, W. M.B. Duval, Solid-Liquid Interface Shape Control During Crystal Growth, Proc. of the Amer. Control Conf., Seattle, WA, 1995, 1270-1274

[16] E. Shaqfeh, C. Jurgensen, Simulation of Reactive Ion Etching Pattern Transfer, J. Appl. Phys. 66, 1989, 4664-4675

[17] V. Singh, E. Shaqfeh, J. McVittie, Simulation of Profile Evolution in Silicon reactive Ion Etching with Re-emission and Surface Diffusion, J. Vac. Technol. B10, 1989, 1091-1104

[18] D. Adalsteinsson, J. A. Sethian, A level Set Approach to a Unified Model for Etching, Deposition, and Lithography I: Algorithms and Two-Dimensional Simulations, J. of Comp. Phys. 120, 1995, 128-144

[19] G. A. Sod, Numerical Methods in Fluid Dynamics, Cambridge University Press, Cambridge, 1985

[20] R.. J. LeVeque, Numerical Methods for Conservation Laws, Birkhauser, Boston, 1992

[21] S. J.. Osher, J. A. Sethian, Fronts Propagation with Curvature dependent Speed: Algorithms based on Hamilton-Jacobi Formulations, J. of Comp. Phys. 79, 1988, 12-49

3.

\subsection{Student Support}

\subsubsection{Academic Year support}

Demarron Berkley - Senior engineering major

1. Timothy Coffee - Junior mathematics major

Noel Gardner - Junior mathematics major

Taurus Smith - Junior mathematics major

Duane Thomas- Junior mathematics major

Sasha Watkins-Senior mathematics major 
Mohammad Aziz-Junior engineering major

Clarissa Zellars- Senior mathematics major

\subsubsection{Summer Support}

Darryl Corey-Graduate Student in Computational Science

Teresa Hodge-Graduate Student in Computational Science

Alice Livingston-Graduate Student in Computational Science

Orlando Burch-Senior mathematics major

1. Mannietea Curry-Senior mathematics major

Alex Serieux-Senior engineering major

Kyron Williams-Senior physics major

Clarissa Zellars- Senior mathematics major

\section{Appendix}




\title{
Temperature Control In Polymer Extrusion Processes $\uparrow$
}

\author{
Max D. Gunzburger
}

L. Steven Hou

James C. Turner

\begin{abstract}
A bstract. An optimization problem is formulated motivated by the desire to obtain uniform extrudate temperature at the dic exit in a polymer extrusion process. Control is effected by adjustments to the heat flux alung the surface of the pipe. An optimality system of partial differential equations is derived from which optimal contruls and states may be deterumind. Then, finite clement discretizations of the optimality system are defined and error estimates are provided along with an eflicient solution algorithm for the discrete equations. Finally, computational results are given for a model example with Oldroyd type Huid, demonstrating the elfectiveness of our theory and method as well as their potential applicability to industrial problems.
\end{abstract}

\section{Introduction}

In polyıner extrusion processes, one is often interested in maintaining a quasi-uniform temperature, so as to reduce material (extrusion product) inhomogeneity, throughout the extrudate cooling process. In this paper, we will study the somewhat simplified case: one tries to obtain a uniform temperature distribution at the exit under steady state situation. The means we use to achieve such a uniform temperature distribution at the exit is to adjust the heat flux on the surface of the pipe near the exit.

'The extrudate in question will be assumed to be viscoelastic fluid of Oldroyd type such as polymer melts wilh a fast relaxation mode [10]. Let $\mathbf{u}$ denote the velocity field, $p$ the pressure field, $T$ the temperature field and $\boldsymbol{\tau}$ the purely elastic part of the extra stress. Let $\mathbf{D}=\frac{1}{2}\left(\operatorname{grad} \mathbf{u}+\operatorname{grad} \mathbf{u}^{T}\right), \mathbf{W}=\frac{1}{2}\left(\operatorname{grad} \mathbf{u}-\operatorname{grad} \mathbf{u}^{T}\right)$ and $D_{a} \tau=(\mathbf{u} \cdot \mathrm{grad}) \tau+\tau \mathbf{W}-\mathbf{W} \tau-a(\mathbf{D} \tau+\boldsymbol{r D})$ where $-1 \leq a \leq 1$. The parameters $R e, W e$ and $\omega$ are the Reynolds number, Weissenberg number and retardation parameter, respectively. The governing equations fur the Oldroyd type fluid, in dimensionless form, is given by the Navier-Stokes equations

$$
\operatorname{Re}(\mathbf{u} \cdot \operatorname{grad}) \mathbf{u}+\operatorname{grad} p=(1-\omega) \Delta \mathbf{u}+\operatorname{div} \mathbf{\tau}+\mathbf{f}, \quad \text { in } \Omega,
$$

the incompressiblity constraint

$$
\operatorname{div} \mathbf{u}=0 \text { in } \Omega .
$$


the constitutive equation (Oldroyd model)

$$
\boldsymbol{\tau}+W e D_{a} \tau=2 \omega \mathrm{D} \text { in } \Omega
$$

and, for simplicity, the boundary condition

$$
\mathbf{u}=\mathbf{h} \text { on } \Gamma \text {, }
$$

and also the energy equation

$$
\begin{aligned}
& -\kappa \Delta T+(\mathbf{u} \cdot \operatorname{grad}) T \\
& =\bar{Q}+2 \mu\left(\operatorname{grad} \mathbf{u}+\operatorname{grad} \mathbf{u}^{T}\right):\left(\operatorname{grad} \mathbf{u}+\operatorname{grad} \mathbf{u}^{T}\right) \text { in } \Omega,
\end{aligned}
$$

with boundary conditions

$$
\begin{gathered}
T=0 \text { on } \Gamma_{D}, \\
\frac{\partial T}{\partial n}=H_{N} \text { on } \Gamma_{N} \cup \Gamma_{O}, \\
\frac{\partial T}{\partial n}=g \text { on } \Gamma_{C} .
\end{gathered}
$$

The data functions $\mathbf{f}, \bar{Q}, H_{N}$, and $\mathbf{h}$ are assumed known; the control $g$ is to be determined so that hot spots are avoided. The constants $\kappa$ and $\mu$ depend on the thermal conductivity coefficient, density, specific heat at constant volume, and viscosity coefficient of the fluid. See [11] for details. We assumed that buoyancy effects can be negelected, and thus the temperature variable does not appear in (1.1).

Two means of obtaining a uniform temperature distribution come to mind. The first is to make the gradient of the temperature along the boundary $\Gamma_{o}$ small. Thus, for example, given a velocity field $u$, we would seek a temperature field $T$ and a control field $g$ such that the functional

$$
\mathcal{M}(T, g)=\frac{\alpha}{2} \int_{\Gamma_{o}}\left|\operatorname{grad}_{s} T\right|^{2} d \Gamma+\frac{\kappa \delta}{2} \int_{\Gamma_{c}}|g|^{2} d \Gamma
$$

is minimized subject, of course, to the constraints imposed by the flow equations (1.5)-(1.8). Here, grad, denotes the surface gradient operator, e.g., in $\mathbf{R}^{2}$, the tangential derivative operator $\partial / \partial \tau$. The non-negative parameters $\alpha$ and $\delta$ can be used to change the relative importance of the the two terms appearing in the definition of $\mathcal{M}$ as well as to act as penalty parameters. The appearance of the control $g$ in the definition of $\mathcal{J}$ is necessary because we are not imposing any a priori limits on the size of this control. The minimization of (1.9) results in a quasi-uniform temperature distribution along the boundary segment $\Gamma_{0}$ because the surface derivatives of the temperature are forced to be small. Another means of achieving the desired result is to try to directly force the temperature field itself to be quasi-uniform. Thus, now, given a velocity field $\mathbf{u}$, we would seek a temperature field $T$ and a control field $g$ such that the functional

$$
\mathcal{J}(T, g)=\frac{1}{2 \gamma} \int_{\Gamma_{o}}\left|T-T_{d}\right|^{2} d \Gamma+\frac{\kappa \delta}{2} \int_{\Gamma_{C}}|g|^{2} d \Gamma
$$

is minimized subject to (1.1)-(1.8), where $T_{d}$ is some desired temperature distribution, e.g., something close to the average temperature along $\Gamma_{C}$ for the uncontrolled system. The non-negative parameters $\gamma$ and $\delta$ can be used to change the relative importance of the the two terms appearing in the definition of $\mathcal{J}$ as well as to act as penalty parameters. As will be demonstrated by numerical examples in $\S 6$, a small $\gamma$ is more useful in achieving quasi-uniform boundary temperature distributions, although it also reduces the accuracy of the 
approximate solution. We will examine the latter issue in $\$ 4$. Numerical experiments show that both (1.9) and (1.10) work effectively for the desired objective. We will focus our discussion on (1.10) thoroughout this paper.

Under the realistic assumption that $\mathbf{u} \cdot \mathbf{n}=0$ on $\Gamma_{C} \cup \Gamma_{N}$, we may prove the existence and uniqueness of optimal solutions and derive an optimality system, i.e., a set of equations from which the optimal control and state may be determined. Also, finite element methods are used to compute an approximate solution of the optimality system. Optimal error estimates are derived and numerical examples are presented. We have also developed an iterative algorithm to compute the approximate solution. The convergence of our algorithm is proved and a comparison with the direct method is made.

Control problems for the fully coupled problem as well as temperature matching for the entire extrudate will be addressed elsewhere.

We close this section by introducing some of the notation used in subsequent sections. Throughout, $C$ will denote a positive constant whose meaning and value changes with context. Also, $H^{s}(\mathcal{D}), s \in \mathbf{R}$, denotes the standard Sobolev space of order $s$ with respect to the set $\mathcal{D}$, where $\mathcal{D}$ is either the flow domain $\Omega$, or its boundary $\Gamma$, or part of that boundary. Of course, $H^{0}(\mathcal{D})=L^{2}(\mathcal{D})$. Dual spaces will be denoted by $(\cdot)^{*}$. Of particular interest will be the space

$$
H_{D}^{1}(\Omega)=\left\{S \in H^{1}(\Omega) \quad: \quad S=0 \text { on } \Gamma_{D}\right\}
$$

Norms of functions belonging to $H^{s}(\Omega), H^{s}(\Gamma)$ and $H^{s}\left(\Gamma_{C}\right)$ are denoted by $\|\cdot\|_{s},\|\cdot\|_{s, \Gamma}$ and $\|\cdot\|_{s, \Gamma_{c}}$, respectively.

The inner product in $L^{2}(\Omega)$ is denoted by $(\cdot, \cdot)$, that in $L^{2}(\Gamma)$ by $(\cdot, \cdot)_{\Gamma}$, that in $L^{2}\left(\Gamma_{O}\right)$ by $(\cdot, \cdot) r_{o}$, and that in $L^{2}\left(\Gamma_{C}\right)$ by $(\cdot, \cdot)_{\Gamma_{C}}$. Since, in general, we will use $L^{2}$-spaces as pivot spaces, these notation will also be employed to denote pairings between Sobolev spaces and their duals.

We will use the bilinear form

$$
a(T, S)=\int_{\Omega} \operatorname{grad} T \cdot \operatorname{grad} S d \Omega \quad \forall T, S \in H^{1}(\Omega)
$$

and the trilinear form

$$
c(\mathbf{u}, T, S)=\int_{\Omega}(\mathbf{u} \cdot \operatorname{grad} T) S d \Omega \quad \forall \mathbf{u} \in \mathbf{H}^{1}(\Omega) \text { and } \forall T, S \in H^{1}(\Omega) .
$$

These forms are continuous in the sense that there exist constants $c_{a}$ and $c_{c}>0$ such that

$$
|a(T, S)| \leq c_{a}\|T\|_{1}\|S\|_{1} \quad \forall T, S \in H^{1}(\Omega)
$$

and

$$
|c(\mathbf{u}, T, S)| \leq c_{c}\|\mathbf{u}\|_{1}\|T\|_{1}\|S\|_{1} \quad \forall \mathbf{u} \in \mathbf{H}^{1}(\Omega) \quad \text { and } \quad \forall T, S \in H^{1}(\Omega) .
$$

Moreover, we have the coercivity property

$$
a(T, T) \geq C_{a}\|T\|_{1}^{2} \quad \forall T \in H_{0}^{1}(\Omega)
$$

for some constant $C_{a}>0$.

For details concerning the notation employed and the inequalities (1.11)-(1.13), one may consult, e.g., [1] and [7]. 


\section{The Optimization Problem, Existence of Solutions, And Optimality System}

We begin by giving a precise statement of the optimization problem we consider. We will assume the domain $\Omega$ is a polygon in $\mathbf{R}^{2}$. We first recall that (1.1)-(1.4) uncouples from (1.5)-(1.8). We may solve for $(\mathbf{u}, p \tau)$ from (1.1)-(1.4) once and for all and then plug them into (1.5)-(1.8). Thus the only state variable is $T$, i.e., the temperature field, and the only boundary control variable is $g$. The state and control variables are constrained to satisfy the system (1.5)-(1.8), which we recast into the following weak form: find $T \in H_{D}^{1}(\Omega)$ such that

$$
\kappa a(T, S)+c(\mathbf{u}, T, S)=(Q, S)+\kappa(g, S)_{\Gamma_{C}}+\kappa\left(H_{N}, S\right)_{\Gamma_{N}} \quad \forall S \in H_{D}^{1}(\Omega),
$$

where we have introduced the simplifying notation

$$
Q=\bar{Q}+2 \mu\left(\operatorname{grad} \mathbf{u}+\operatorname{grad} \mathbf{u}^{T}\right):\left(\operatorname{grad} \mathbf{u}+\operatorname{grad} \mathbf{u}^{T}\right)
$$

Note that since we seek $T \in H_{D}^{1}(\Omega)$

$$
T=0 \text { on } \Gamma_{D} \text {. }
$$

Throughout, we will assume that the given velocity field $\mathbf{u}$ is smooth and satisfies

$$
\operatorname{div} \mathbf{u}=0 \quad \text { in } \Omega \quad \text { and } \quad \mathbf{u} \cdot \mathbf{n} \geq 0 \quad \text { a.e. on } \Gamma_{C} \cup \Gamma_{N} .
$$

Under these assumptions, we have the useful relation

$$
c(\mathbf{u}, S, S)=\frac{1}{2} \int_{\Gamma}(\mathbf{u} \cdot \mathbf{n}) S^{2} d \Gamma \geq 0 \quad \forall S \in H^{1}(\Omega)
$$

which can be derived by setting $T=S$ in the following integration by parts formula:

$$
c(\mathbf{u}, T, S)=\int_{\Gamma}(\mathbf{u} \cdot \mathbf{n}) T S d \Gamma-c(\mathbf{u}, S, T) .
$$

For each possible control function $g$, there exists a unique corresponding state function $T$.

Lemma 2.1- For every $g \in L^{2}\left(\Gamma_{C}\right)$, there exists a unique $T \in H_{D}^{1}(\Omega)$ such that $(2.1)$ is satisfied. Moreover,

$$
\|T\|_{1}+\|T\|_{0, \Gamma_{C}} \leq C\left(\|g\|_{0, \Gamma_{C}}+\|Q\|_{0}+\left\|H_{N}\right\|_{0, \Gamma_{N}}\right)
$$

The admissibility set $\mathcal{U}_{a d}$ is defined by

$$
\mathcal{U}_{a d}=\left\{(T, g) \in H_{D}^{1}(\Omega) \times L^{2}\left(\Gamma_{C}\right): \mathcal{J}(T, g)<\infty,(2.1) \text { is satisfied }\right\}
$$

Then, $(\hat{T}, \hat{g}) \in \mathcal{U}_{a d}$ is called an optimal solution if there exists $\epsilon>0$ such that

$$
\mathcal{J}(\hat{T}, \hat{g}) \leq \mathcal{J}(T, g) \quad \forall(T, g) \in \mathcal{U}_{a d} \text { satisfying }\|T-\hat{T}\|_{1}+\|g-\hat{g}\|_{0, \Gamma_{c}} \leq \epsilon .
$$

Based on the previous lemma, we can show the existence and uniqueness of optimal solutions.

Theorem 2.2- There exists a unique optimal solution $(\hat{T}, \hat{g}) \in \mathcal{U}_{a d}$.

Using techniques in e.g. [9] we may obtain the optimality condition

$$
g=-\left.\frac{1}{\delta} \Phi\right|_{\Gamma_{c}}
$$


where $\Phi$ is the solution of the adjoint state equation

$$
\kappa a(R, \Phi)+c(\mathbf{u}, R, \Phi)-\frac{1}{\gamma}\left(R, T-T_{d}\right)_{\Gamma_{c}}=0 \quad \forall R \in H_{D}^{1}(\Omega)
$$

Eliminating $g$ from (2.1) and combining with (2.9), we obtain the optimality system

$$
\kappa a(T, S)+c(\mathbf{u}, T, S)+\frac{\kappa}{\delta}(\Phi, S)_{\Gamma_{C}}=(Q, S)+\kappa\left(H_{N}, S\right)_{\Gamma_{N}} \quad \forall S \in H_{D}^{1}(\Omega)
$$

and

$$
\kappa a(R, \Phi)+c(\mathbf{u}, R, \Phi)-\frac{1}{\gamma}\left(R, T-T_{d}\right)_{\Gamma_{c}}=0 \quad \forall R \in H_{D}^{1}(\Omega) .
$$

Thus, the optimal state, i.e., the temperature distribution $T$, can be found by solving the coupled system (2.10)-(2.11), which also provides the optimal co-state $\Phi$. The optimal control $g$ can then be deduced from (2.8).

\section{Finite Element Approximation, Error Estimates, and Iterative Methods}

In the usual manner, one may construct finite element subspaces $W^{h} \subset H_{D}^{1}(\Omega) \cap C(\bar{\Omega})$ parametrized by a parameter $h$ that tends to zero. (In practice, $h$ is, of course, related to a grid size.) We assume the approximation property (see [3]): there exist an integer $k$ and a constant $C$ such that

$$
\inf _{S^{\Lambda} \in W^{\star}}\left\|S-S^{h}\right\|_{1} \leq C h^{m}\|S\|_{m+1} \quad \forall S \in H_{D}^{1}(\Omega) \text { and } 0 \leq m \leq k .
$$

A finite element algorithm for determining approximations of the solution of the optimality system (2.9)(2.10) is then defined as follows: seek $T^{h} \in W^{h}$ and $\Phi^{h} \in W^{h}$ such that

$$
\begin{aligned}
\kappa a\left(T^{h}, S^{h}\right)+c\left(\mathbf{u}, T^{h}, S^{h}\right)+\frac{\kappa}{\delta}\left(\Phi^{h}, S^{h}\right) \Gamma_{c} & \\
& =\left(Q, S^{h}\right)+\kappa\left(H_{N}, S^{h}\right) \Gamma_{N} \quad \forall S^{h} \in W^{h}
\end{aligned}
$$

and

$$
\kappa a\left(R^{h}, \Phi^{h}\right)+c\left(\mathbf{u}, R^{h}, \Phi^{h}\right)-\frac{1}{\gamma}\left(R^{h}, T^{h}-T_{d}\right)_{\Gamma_{c}}=0 \quad \forall R^{h} \in W^{h} .
$$

Although the optimality system is linear, the coupling of $\mathbf{u}$ and $\Phi$ in the two equations make the derivation of error estimates nontrivial. It turns out to be convenient to apply the Brezzi-Rappaz-Raviart theory (see [2], [5], and [7]) to obtain error estimates.

Theorem 3.1-Let $(T, \Phi)$ and $\left(T^{h}, \Phi^{h}\right)$ be the solutions of (2.9)-(2.10), and (3.2)-(3.3), respectively. Assume that $T, \Phi \in H^{m+1}(\Omega) \cap H_{D}^{1}(\Omega)$ for some $1 \leq m \leq k$; also assume that (3.1) holds. Then,

$$
\begin{aligned}
\| T- & T^{h}\left\|_{1}+\right\| \Phi-\Phi^{h} \|_{1} \\
& \leq C \max \left\{\frac{1}{\delta}, \frac{1}{\gamma}, 1\right\} h^{m}\left(\|Q\|_{m-1}+\left\|H_{N}\right\|_{\Gamma_{N}, m-1 / 2}+\left\|T_{d}\right\|_{\Gamma_{C}, m-1 / 2}\right)
\end{aligned}
$$

where $C$ is independent of $h, \delta, \gamma, T$, and $\Phi$.

A simple iterative algorithm for solving (2.10)-(2.11) can be defined as follows:

$$
\text { choose } \Phi^{(0)}
$$


for $n=1,2, \ldots$, solve for $T^{(n)}$ from

$$
\begin{aligned}
& \kappa a\left(T^{(n)}, S\right)+c\left(u, T^{(n)}, S\right) \\
& \quad=-\frac{\kappa}{\delta}\left(\Phi^{(n-1)}, S\right) \Gamma_{c}+(Q, S)+\kappa\left(H_{N}, S\right)_{\Gamma_{N}} \quad \forall S \in H_{D}^{1}(\Omega) ;
\end{aligned}
$$

then solve for $\Phi^{(n)}$ from

$$
\kappa a\left(R, \Phi^{(n)}\right)+c\left(u, R, \Phi^{(n)}\right)=\frac{1}{\gamma}\left(R, T^{(n)}-T_{d}\right) \Gamma_{c} \quad \forall R \in H_{D}^{1}(\Omega) .
$$

Of course, ultimately, this algorithm has to be carried out in a discretized version, such as one using a finite element method.

The convergence of this algorithm can be proved as a result of the observation that it is effectively a gradient method for the following minimization problem: find $g \in L^{2}(\Gamma c)$ such that $\mathcal{K}(g):=\mathcal{J}(T(g), g)$ is minimized where $T(g) \in H_{D}^{1}(\Omega)$ is defined as the solution of (2.1).

Theorem 5.2- Let $\left(T^{(n)}, \Phi^{(n)}\right)$ be the solution of (3.4)-(3.5) and $(T, \Phi)$ the solution of $(2.9)-(2.10)$. Then, $T^{(n)} \rightarrow T$ in $H_{D}^{1}(\Omega)$ and $\Phi^{(n)} \rightarrow \Phi$ in $H_{D}^{1}(\Omega)$ as $n \rightarrow \infty$.

\section{Computational Examples}

Let $\Omega \subset \mathbb{R}^{2}$ be the unit square $(0,1) \times(0,1)$. Let $\Gamma=\Gamma_{C} \cup \Gamma_{D} \cup \Gamma_{N} \cup \Gamma_{0}$ be shown as in Figure 1 .

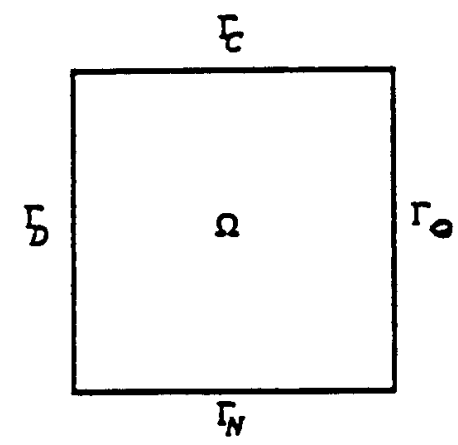

Figure 1. Computational domain

The finite element spaces $W^{h}$ are chosen to be piecewise linear elements on a triangular mesh. All the numerical results make use of the following parameters and data:

$$
\begin{aligned}
& \text { parameters: } \begin{array}{r}
\kappa=1 / 0.73 ; \quad R e=1 ; \quad W e=1 ; \omega=1 / 2 ; \\
\text { boundary data: } \quad T=1 \text { on } \Gamma_{D}, \quad \frac{\partial T}{\partial n}=0 \text { on } \Gamma_{N} \cup \Gamma_{O} ; \\
\text { heat source: } \quad Q=-\frac{5 \kappa}{2}\left(9 \pi^{2} \cos (3 \pi x) \cos ^{2}(\pi y)-4 \pi^{2} \sin ^{2}\left(\frac{3}{2} \pi x\right) \cos (2 \pi y)\right) \\
+\left(1-y^{2}\right) \frac{15 \pi}{2} \sin (3 \pi x) \cos ^{2}(\pi y) ;
\end{array}
\end{aligned}
$$


velocity profile:

elastic extra stress profile:

pressure profile:

In functional (1.10), we choose

$\mathbf{u}=\left(1-y^{2}, 0\right)$

$$
\tau=\left(\begin{array}{cc}
0 & -y \\
-y & -4 y^{2}
\end{array}\right)
$$

$$
p=4\left(1-y^{2}\right)-x
$$

$$
T_{d}=3.5 \text {. }
$$

For the data given above, the exact solution of the uncontroled problem, i.e., for

$$
\frac{\partial T}{\partial n}=0 \text { on } \Gamma_{C}
$$

is given by $T=5 \sin ^{2}\left(\frac{3}{2} \pi x\right) \cos ^{2}(\pi y)+1$.

We compare the temperature distribution in the uncontroled case with the optimal temperature distribution in the controled case for which

$$
\frac{\partial T}{\partial n}=g \quad \text { on } \Gamma_{c}
$$

where $g$ is the control such that (1.10) is minimized. Approximations to the optimal state and co-state are computed from (3.2)-(3.3); the approximate optimal control $g^{h}$ is then obtained from (2.8), i.e., $g^{h}=$ $-\left.(1 / \delta) \Phi^{h}\right|_{c}$. All of the computational results shown below were obtained with the use of a mesh size $h=\frac{1}{19}$. Of course, calculations with varying mesh sizes were performed. Since these merely verified the error estimates, we do not report on them here.

Specifically, Figures $2-4$ deal with the following cases:

1. Exact, uncontroled temperature and; Optimal temperature;

2. Exact, uncontroled temperature and; Optimal temperature;

2. Optimal boundary control.
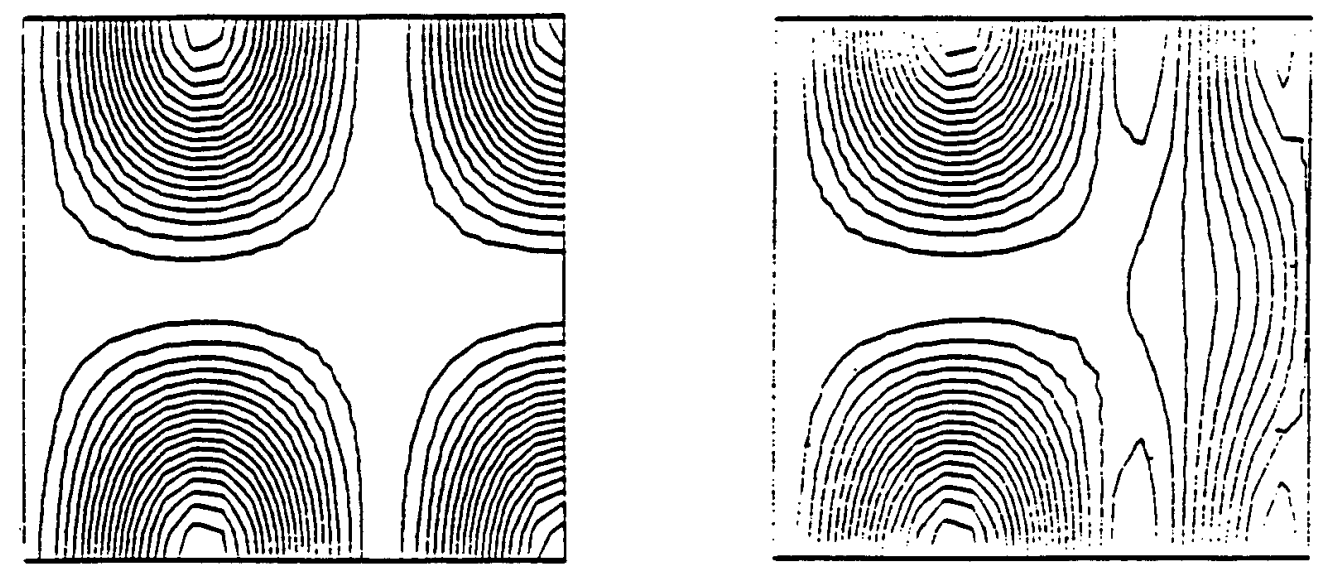

Figure 2. Temperature contours: uncontroled and controled.

Optimal boundary control on $\Gamma_{C}$.

( $\Gamma_{C}$ is the top boundary segment.)

( $\Gamma_{0}$ is the right boundary segment.) 

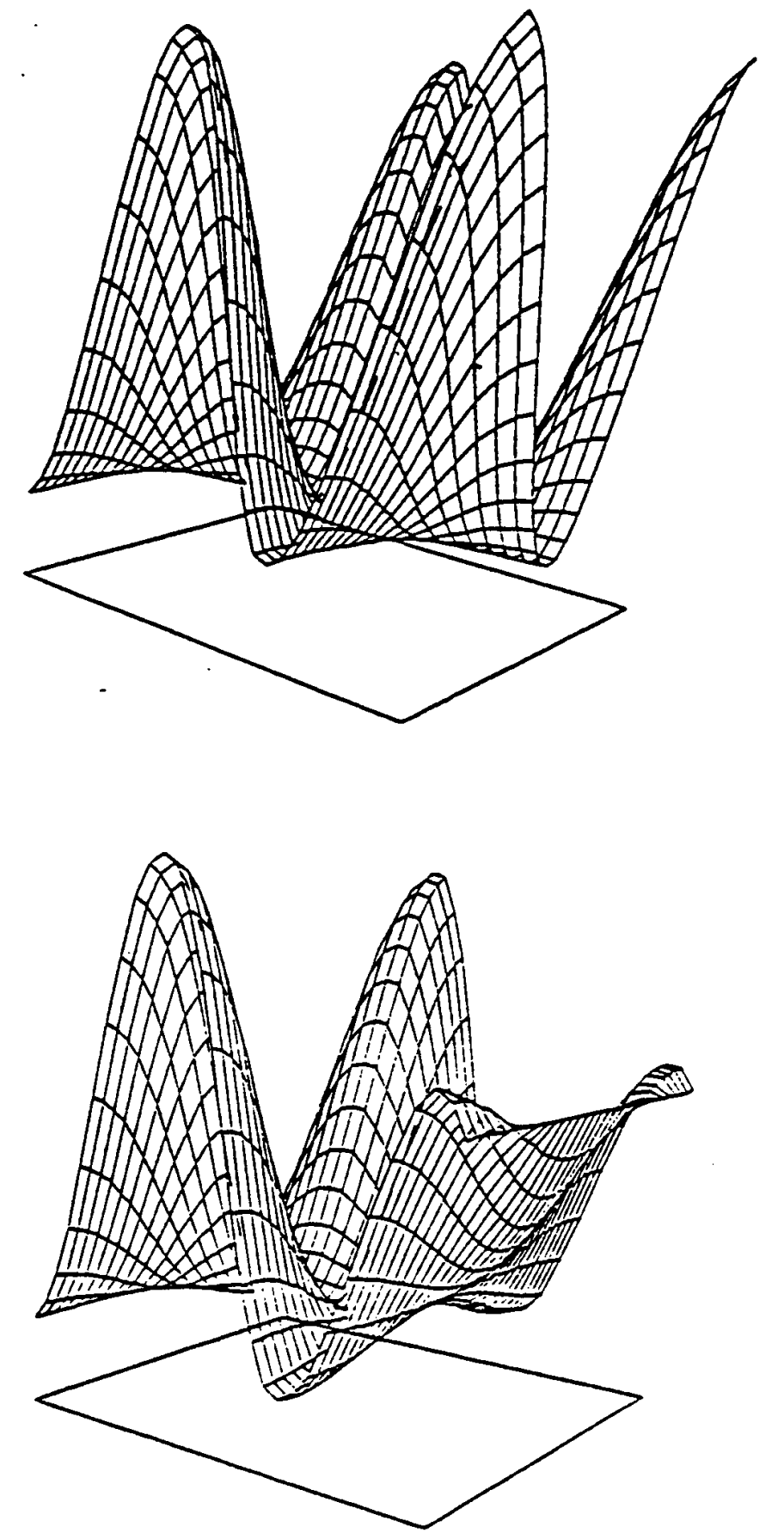

Figure 3. Temperature surfaces: uncontroled and controled.

( $\Gamma_{C}$ is the top-right boundary segment.)

( $\Gamma_{0}$ is the lower-right boundary segment.) 


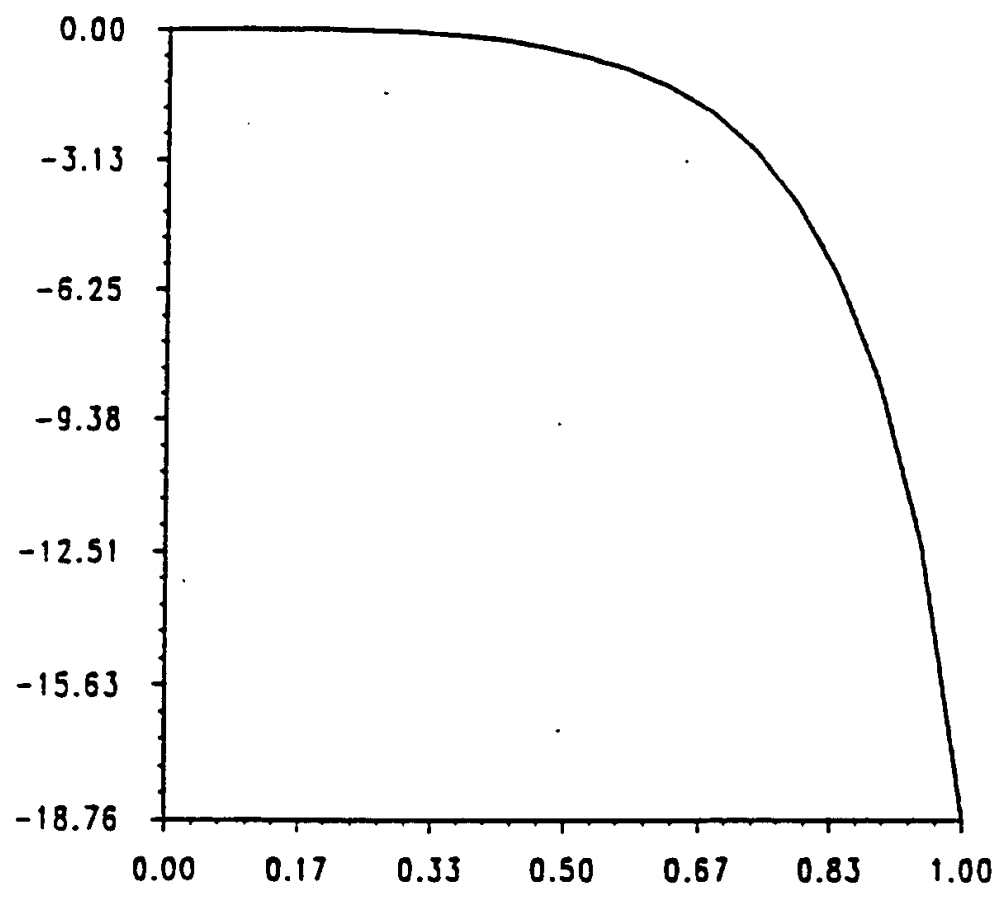

Figure 4. Optimal boundary control on $\Gamma_{C}$.

References

[1] R. ADAMS, Sobolev Spaces, Academic, New York, 1975.

[2] F. BREzzI, J. RAPPAZ and P.-A. RAVIART, Finite-dimensional approximation of nonlinear problems. Part I: branches of nonsingular solutions, Numer. Math. 36 1980, 1-25.

[3] P. Clarlet, The Finite Element Method for Elliptic Problems, North-Bolland, Amsterdam, 1978.

[4] P. CiARLET, Introduction to Numerical Linear Algebre and Optimisation, Cambridge, Cambridge, 1989.

[5] M. CRouzleX and J. RAPPAz, On Numerical Approximation in Bifurcation Theory, Masson, Paris, 1989.

[6] P. CuVELIER, Optimal control of a system governed by the Navier-Stokes equations coupled with the heat equations, New Developments in Differential equations (Ed. by W. Eckhaus), North-Holland, Amsterdam, 1976, 81-98.

[7] V. Grault and P.-A. Raviart, Finite Element Methods for Navier-Stokes Equations, Springer, Berlin, 1986.

[8] M. GUNZBURGer, L. HOU and T. SvOBODNY, Control of temperature distributions along boundaries of engine components, Numerical Methods for Laminar and Turbulent Flows - Proceedings. of the 7th International Conference on Numerical Methods for Turbulent and Laminar Flows, Stanford, July, 1991.

[9] J.-L. Lions Contröl Optimal de Systèmes Gouvernés par des Equations aux Dérivees Partielles, Dunod, Paris, 1968.

[10] J.G. OLDnoyd, On the formation of rheological equations of state, Proc. Roy. Soc. London, A 200, 1950, 523-541.

[11] J. Serrin, Mathematical principles of classical fluid mechanics, Bandbüch der Physik VIII/1 (ed. by S. Flügge and C. Truesdell), Springer, Berlin, 1959, 125-263. 


\title{
A New Condition of the Exact Boundary Controllability for Parabolic Equations
}

\author{
Yanzhao Cao, Max Gunzburger and James Turner
}

April 26, 1997

\section{Introduction}

The purpose of this paper is to investigate the exact controllability problems for systems modeled by linear parabolic differential equations. This problem has been studied extensively in the last 30 years. A very significant early contribution is due to Egorov ([2], [3]). Another early paper is due to Gal'chuk ([9]). Many developments in the controllability theory of the linear parabolic equations and hyperbolic equations are due to Fattorini and Russell ([4], [5], [19], [20]). Using a harmonic analysis method, they obtained results such as approximate controllability and conditions on $\hat{y}$ for the exact controllability of systems governed by hyperbolic and parabolic equations. We refer to [7], [8] for more recent development by Furskov and Imanuvilov.

In this paper we attempt to study the exact controllability problem by examining the limit behavior of the corresponding optimal control problem. Using this method we obtained a new condition on the exact controllability of the system. We believe that this condition is at least comparible condition obtained in [18]. The paper is organized as follows. In $\S 2$, we state the problem and some results concerning linear parabolic differential equations. In $\S 3$ we give a constructive proof for the approximate controllability of the system using our method. Finally in $\S 4$ we give a condition on the exact controllability of the system. 


\section{Statement of the Problem, Notation and Preliminaries}

Let $\Omega$ be a bounded domain in $\mathrm{R}^{\mathrm{d}}$. For a fixed $T$, let $Q=[0, T] \times \Omega$ and $\Sigma=(0, T) \times \partial \Omega$. On the domain $\Omega$, let $A$ be the second order elliptic differential operator

$$
A y=-\sum_{i, j=1}^{d} \frac{\partial}{\partial x_{i}}\left(a_{i, j}(x) \frac{\partial y}{\partial x_{j}}\right)+c(x) y
$$

We will assume that $c>0$ on $\bar{\Omega}$ and that the matrix $\left(a_{i, j}(x)\right)$ is symmetric and positive definite.

Consider now the parabolic initial boundary value problem

$$
\begin{aligned}
\frac{\partial y}{\partial t}+A y & =0, \quad \text { in } Q \\
\frac{\partial y}{\partial \nu} & =u, \quad \text { on } \Sigma \\
y(0) & =y_{0}, \quad \text { in } \Omega \text { for } t=0 .
\end{aligned}
$$

Here $\frac{\partial}{\partial \nu}=\sum_{i, j=1}^{d} a_{i, j} n_{i}\left(\frac{\partial}{\partial x_{j}}\right)$ where $n_{i}$ is the $i-t h$ component of the outward unit normal on $\partial \Omega$. We say that the system is exactly controllable for a given state $\hat{y}$ if there exists $u \in L^{2}(\Sigma)$ such that the solution $y$ of problem (2.1) also satisfies

$$
y(T, \cdot)=\hat{y}
$$

Define a cost functional

$$
J_{\epsilon}(u)=\frac{1}{2} \int_{\Omega}(y(T, x ; u)-\widehat{y}(x))^{2} d x+\frac{\epsilon}{2} \int_{\Sigma} u^{2}(t, x) d x d t
$$

where $y=y(t, x ; u)$ satisfies $(2.1) . u_{\epsilon}(t, x)$ is said to be an optimal control if it satisfies

$$
J_{\epsilon}\left(u_{\epsilon}\right)=\inf _{u \in L^{2}(Q)} J_{\epsilon}(u)
$$

For a Banach space $X$, define

$$
L^{2}(0, T ; X)=\left\{f:(0, T) \rightarrow X ; \int_{0}^{T}\|f(t)\|_{X}^{2} d t<\infty\right\}
$$

It is a Hilbert space with the norm

$$
\|f\|_{L^{2}(0, T ; X)}=\left(\int_{0}^{T}\|f(t)\|_{X}^{2} d t\right)^{\frac{1}{2}}
$$

Let $L^{2}(\Omega)$ and $L^{2}(\partial \Omega)$ be the usual $L^{2}$ function spaces. We shall use the notation

$$
(\phi, \psi)=\int_{\Omega} \phi \psi d x
$$


and

$$
<\phi, \psi>=\int_{\partial \Omega} \phi \psi d \omega
$$

for the inner products and the associated norms will be denoted by $\|\cdot\|$ and $|\cdot|$, respectively. We also define

$$
W(0, T)=\left\{f ; f \in L^{2}\left(0, T ; H^{1}(\Omega)\right), \frac{d f}{d t} \in L^{2}\left(0, T ; H^{-1}(\Omega)\right)\right\}
$$

where $\frac{d}{d t}$ is taken in the sense of distributions on $(0, T)$ with values in $H^{1}(\Omega) . W(0, T)$ is a Hilbert space with the norm

$$
\|f\|_{W(0, T)}=\left(\|f\|_{L^{2}\left(0, T ; H^{1}(\Omega)\right)}^{2}+\left\|\frac{d f}{d t}\right\|_{L^{2}\left(0, T ; H^{-1}(\Omega)\right.}^{2}\right)^{\frac{1}{2}} .
$$

For $p, q>0$, let also

$$
H^{p, q}(Q)=L^{2}\left(0, T ; H^{q}(\Omega)\right) \cap H^{p}\left(0, T ; L^{2}(\Omega)\right)
$$

These spaces are described in [15], and their norms are defined by

$$
\|f\|_{H^{p, q}(Q)}=\left(\|f\|_{L^{2}\left(0, T ; H^{q}(\Omega)\right)}^{2}+\|f\|_{H^{p}\left(0, T ; L^{2}(\Omega)\right)}^{2}\right)^{\frac{1}{2}}
$$

The spaces $H^{p, q}(\Sigma)$ with associated norms $\|\cdot\|_{H^{p, q}(\Sigma)}$ are defined similarly by replacing $\Omega$ by $\Sigma$ above.

We recall that if $q>\frac{1}{2}$ and $p=q-\frac{1}{2}$, then there is a constant $c$ such that for any $f \in H^{p, \frac{p}{2}}(Q)$,

$$
\|f\|_{H^{p, \frac{p}{2}(\Sigma)}} \leq C\|f\|_{H^{q, q} \frac{q}{2}(Q)}
$$

and if $q>1, g \in H^{q, \frac{q}{2}}(\Sigma)$ and $t \in[0, T]$ then

$$
\|f(t)\|_{H^{p-1}(\Omega)} \leq c\|f\|_{H^{p, \frac{p}{2}(Q)}}
$$

and

$$
|g|_{H^{p-1}(\partial \Omega)} \leq c\|g\|_{H^{q, \frac{q}{2}}(\Sigma)} .
$$

Define the bilinear form $a: H^{1}(\Omega) \times H^{1}(\Omega) \rightarrow R$ by

$$
a(\phi, \psi)=\int_{\Omega}\left\{\sum_{i, j=1}^{d} a_{i j}(x) \frac{\partial \phi}{\partial x_{i}} \frac{\partial \psi}{\partial x_{j}}+c(x) \phi \psi\right\} d x .
$$

Note that from the properties of the operator $A$ we have that there exist constants $c_{1}, c_{2}>0$ such that

$$
|a(\phi, \psi)| \leq c_{1}\|\phi\|_{1}\|\psi\|_{1}
$$


and

$$
a(\phi, \phi) \geq c_{2}\|\phi\|_{1}^{2}
$$

for all $\phi, \psi \in H^{1}(\Omega)$.

We now define the operators for weak solutions of elliptic boundary value problems. For $f \in H^{-1}(\Omega)$, let $T f \in H^{1}(\Omega)$ denote the unique solution of the problem

$$
a(T f, \phi)=(f, \phi) \quad \text { for } \phi \in H^{1}(\Omega) .
$$

$T$ is a linear operator on $H^{-1}(\Omega)$. Also since

$$
(T f, \phi)=a(T f, T \phi)=(f, T \phi)
$$

for any $f, \phi \in L^{2}(\Omega), T$ is self adjoint on $L^{2}(\Omega)$. Next we introduce the eigenvalue problem

$$
\begin{aligned}
& A \varphi=\Lambda \varphi, \quad \text { in } \Omega, \\
& \frac{\partial \varphi}{\partial \nu}=0, \quad \text { on } \partial \Omega .
\end{aligned}
$$

It is well known that this problem has a system of eigenfunctions $\left\{\varphi_{j}\right\}_{j=1}^{\infty}$, forming a complete orthonormal set in $L^{2}(\Omega)$, with eigenvalues $0<\lambda_{1} \leq \lambda_{2} \leq \cdots \leq \lambda_{j} \rightarrow \infty$ as $j \rightarrow \infty$. Note that if we let $\nu_{j}=\lambda_{j}^{-1}$, then

$$
T \phi_{j}=\nu_{j} \phi_{j} \quad j=1,2, \ldots
$$

W now consider the weak formulation of problem (2.1)

$$
\begin{aligned}
\left(\frac{d y}{d t}, \phi\right)+a(y, \phi) & =\left\langle u, \phi>, \quad \text { for } \phi \in H^{1}(\Omega),\right. \\
y(0, \cdot) & =y_{0} .
\end{aligned}
$$

It is well known that if $y_{0} \in L^{2}(\Omega)$ and $u \in L^{2}(\Sigma)$ then (2.3) has a unique solution $y$ in $W(0, T)$, and there is a constant $C$, independent of $y_{0}$ and $u$, such that

$$
\|y\|_{W(0, T)} \leq C\left(\|v\|+\|u\|_{L^{2}(\Sigma)}\right) .
$$

If $u=0$, then the exact solution of (2.3) can be represented by

$$
y(t, x)=\sum_{j=1}^{\infty}\left(v, \varphi_{j}\right) e^{-\lambda_{j} t} \varphi_{j}(x)
$$

The following lemma can be found in [21].

Lemma 2.1 Let $\phi \in H^{p}(\Omega)$ for $-1 \leq p \leq 1$. Then

$$
\left\||\phi \||=\left(\sum_{j=1}^{\infty}\left(\phi, \varphi_{j}\right)^{2} \lambda^{p}\right)^{\frac{1}{2}}\right.
$$

is an equivalent norm in $H^{p}(\Omega)$. 


\section{Approximate Controllability}

3.1 An operator representation of the terminal state. In the following lemma we state the optimality system for the problem (2.2). It will play a central role in obtaining the main results of this section and the next section. From now on, we suppress the explicit dependence on $x$, e.g., we will use $u(t)$ to mean $u(t, x)$.

Lemma 3.1 ([12]) The optimality system of problem (2.1) is given by

$$
\begin{aligned}
\frac{\partial p_{\epsilon}}{\partial t}-A p_{\epsilon} & =0 & & \text { in } Q, \\
\frac{\partial p_{\epsilon}}{\partial \nu} & =0 & & \text { on } \Sigma, \\
p_{\epsilon} & =y_{\epsilon}-\widehat{y} & & \text { in } \Omega \text { for } t=T, \\
p_{\epsilon} & =-\epsilon u_{\epsilon} & & \text { on } \Sigma .
\end{aligned}
$$

The weak formulation of the optimality system can be written as follows.

$$
\begin{aligned}
\left(\frac{d y_{\epsilon}}{d t}, \phi\right)+a\left(y_{\epsilon}, \phi\right) & =\frac{1}{\epsilon}<p_{\epsilon}, \phi>, \quad \text { for } \phi \in H^{1}(\Omega) \\
-\left(\frac{d p_{\epsilon}}{d t}, \phi\right)+a\left(p_{\epsilon}, \phi\right) & =0, \quad \text { for } \phi \in H^{1}(\Omega) \\
y_{\epsilon}(0) & =y_{0} \\
p_{\epsilon}(T) & =\hat{y}-y_{\epsilon}(T) .
\end{aligned}
$$

To represent the terminal state $y_{\epsilon}(T)$ by an operator, we consider, for a given $z \in L^{2}(\Omega)$, the system

$$
\begin{aligned}
\left(\frac{d y_{1}}{d t}, \phi\right)+a\left(y_{1}, \phi\right) & =<p_{1}, \phi>, \quad \text { for } \phi \in H^{1}(\Omega), \\
-\left(\frac{d p_{1}}{d t}, \phi\right)+a\left(p_{1}, \phi\right) & =0, \quad \text { for } \phi \in H^{1}(\Omega), \\
y_{1}(0) & =0 \\
p_{1}(T) & =z .
\end{aligned}
$$

This system has a unique solution $\left(y_{1}(t), p_{1}(t)\right) \in W(0, T)$.

Let $E(t)$ denote the solution operator for the problem

$$
\begin{aligned}
\frac{\partial w}{\partial t}+A w & =0 & & \text { on } Q \\
\frac{\partial w}{\partial \nu} & =0 & & \text { on } \Sigma, \\
w & =z & & \text { in } \Omega \text { for } t=0,
\end{aligned}
$$

i.e.,

$$
w(t)=E(t) z
$$


We now define an operator $R: L^{2}(\Omega) \rightarrow L^{2}(\Omega)$ by

$$
R z=y_{1}(T)
$$

Comparing (3.2) with (3.3), we have that

$$
\frac{1}{\epsilon} R(y(T)-\hat{y})=-E(T) v+y(T)
$$

or

$$
(\epsilon+R)(y(T)-\hat{y})=\epsilon(\hat{y}-E(T) v)
$$

\subsection{Properties of the operator $R$.}

Lemma 3.2 $R \in L\left(L^{2}(\Omega), H^{1}(\Omega)\right)$. Therefore $R$ is a compact operator in $L^{2}(\Omega)$.

Proof: Consider the mapping

$$
z \rightarrow p_{1}(t, \cdot)=E(T-t) z
$$

We prove that this mapping is continuous from $L^{2}(\Omega)$ into $H^{\frac{1}{2}, 1}(Q)$. By standard theory for parabolic differential equations ([15]) this mapping is continuous from $H^{1}(\Omega)$ into $H^{1,2}(Q)$. If $z \in H^{-1}(\Omega)$ then

$$
\begin{aligned}
\int_{0}^{T}\left\|p_{1}(t, \cdot)\right\|^{2} d t & =\int_{0}^{T} \sum_{j=1}^{\infty}\left(z, \phi_{j}\right)^{2} e^{-2 \lambda_{j}(T-t)} d t \\
& =\frac{1}{2} \sum_{j=1}^{\infty}\left(z, \phi_{j}\right)^{2} \lambda_{j}^{-1}\left(1-e^{-2 \lambda_{j} T}\right) \\
& \leq C\|z\|_{-1}
\end{aligned}
$$

Hence the mapping is continuous from $H^{-1}(\Omega)$ into $L^{2}(Q)$. By interpolation ([1]) we have that $R$ is a continuous mapping from $L^{2}(\Omega)$ to $H^{\frac{1}{2}, 1}(Q)$. By $(3.3)$, we have that $\left.\frac{\partial y_{1}}{\partial \nu}\right|_{\Sigma}=\left.p_{1}\right|_{\Sigma}$. Thus

$$
\|R z\|_{1}=\left\|y_{1}(T)\right\|_{1} \leq C\left\|y_{1}\right\|_{H^{1,2}(Q)} \leq C\|z\|
$$

The proof is complete.

Lemma 3.3 $R$ is symmetric and semi-definite.

Proof: For given $z, z_{0} \in L^{2}(\Omega)$, let $(y, p),\left(y_{0}, p_{0}\right)$ be the corresponding solutions of (3.3), respectively. Then

$$
\left(\frac{d y}{d s}, p_{0}\right)+a\left(y, p_{0}\right)=<p, p_{0}>
$$




$$
\begin{gathered}
\left(\frac{d y_{0}}{d s}, p\right)+a\left(y_{0}, p\right)=<p_{0}, p> \\
a\left(y, p_{0}\right)=\left(y, \frac{d}{d s} p_{0}\right) \\
a\left(y_{0}, p\right)=\left(y_{0}, \frac{d}{d s} p\right)
\end{gathered}
$$

We therefore have that

$$
\left(\frac{d}{d s} y(s), p_{0}(s)\right)+\left(y(s), \frac{d}{d s} p_{0}(s)\right)=\left(\frac{d}{d s} y_{0}(s), p(s)\right)+\left(y_{0}(s), \frac{d}{d s} p(s)\right)
$$

or

$$
\frac{d}{d s}\left(y_{0}(s), p(s)\right)=\frac{d}{d s}\left(y(s), p_{0}(s)\right)
$$

Integrating the above equality from 0 to $T$, noting that $y(0)=y_{0}(0)=0$, we have that

$$
\begin{aligned}
\left(y(T), p_{0}(T)\right) & =\int_{0}^{T} \frac{d}{d s}\left(y(s), p_{0}(s)\right) d s \\
& =\int_{0}^{T} \frac{d}{d s}\left(y_{0}(s) p(s)\right) d s \\
& =\left(y_{0}(T), p(T)\right)
\end{aligned}
$$

or

$$
\left(R z, z_{0}\right)=\left(R z_{0}, z\right)
$$

Thus $R$ is self-adjoint. We also have that

$$
\begin{aligned}
|p|^{2} & =\left(\frac{d}{d s} y, p\right)+a(y, p) \\
& =\left(\frac{d}{d s} y, p\right)+\left(y, \frac{d}{d s} p\right) \\
& =\frac{d}{d s}(y, p) .
\end{aligned}
$$

Integrating the above equality, we have that

$$
(R z, z)=\int_{0}^{T}|p|^{2} d s \geq 0 .
$$

So $R$ is semi-definite. The proof is complete.

Lemma 3.4 Assume that $\Sigma$ is analytic. Then $\operatorname{Ker} R=0$.

Proof: Under the given assumption we know that $p$ is analytic in $Q$ and on $\Sigma$. Assume that $R z=0$. Then from the proof of Lemma 3.3 we have that

$$
0=(R z, z)=\int_{0}^{T}|p|^{2} d s
$$


Thus $p=0$ on $\Sigma$. Hence the data corresponding to the Cauchy problem is zero on $\Sigma$ which implies according to the Cauchy-Kowaleska theorem (since $p$ is analytic) that $p=0$ on $Q$. In particular $z=p(T)=0$. The proof is complete.

\subsection{Approximate Controllability}

Theorem 3.5 Let $\hat{y} \in L^{2}(\Omega)$ and $\left(y_{\epsilon}, u_{\epsilon}\right)=\left(y_{\epsilon}(t), u_{\epsilon}(t)\right)$ be the solution of the optimality stystem. Then

$$
\lim _{\epsilon \rightarrow 0}\left\|y_{\epsilon}(T)-\hat{y}\right\|_{L^{2}(\Omega)}=0
$$

Proof: By Lemma 3.2, Lemma 3.3 and Lemma 3.4, and the Gilbert-Schmidt theorem, the operator $R$ has a system of eigenfunctions $\left\{e_{j}\right\}_{j=1}^{\infty}$ with eigenvalues $\lambda_{1} \geq \lambda_{2} \geq \cdots \geq \lambda_{j} \rightarrow 0$ as $j \rightarrow \infty$. Moreover, $\left\{e_{j}\right\}_{j=1}^{\infty}$ forms an orthonormal basis in $L^{2}(\Omega)$. For $\hat{y} \in L^{2}(\Omega)$ let

$$
\hat{y}-E(T) v=\sum_{j=1}^{\infty} y_{j} e_{j}
$$

Then

$$
R(\hat{y}-E(T) v)=\sum_{j=1}^{\infty} \lambda_{j} y_{j} e_{j}
$$

Using (3.6) we have that

$$
y_{\epsilon}(T)-\hat{y}=\sum_{j=1}^{\infty} \frac{\epsilon y_{j} e_{j}}{\epsilon+\lambda_{j}}
$$

Thus

$$
\begin{aligned}
\left\|y_{\epsilon}(T)-\hat{y}\right\|_{L^{2}(\Omega)}^{2} & =\sum_{j=1}^{\infty} \frac{\epsilon^{2} y_{j}^{2}}{\left(\epsilon+\lambda_{j}\right)^{2}} \\
& \leq \sum_{j=1}^{N} \frac{\epsilon^{2} y_{j}^{2}}{\left(\epsilon+\lambda_{j}\right)^{2}}+\sum_{j=N+1}^{\infty} y_{j}^{2} .
\end{aligned}
$$

For any given $\delta>0$, there exists $N$ such that

$$
\sum_{j=N+1}^{\infty} y_{j}^{2}<\frac{\delta}{2} .
$$

For this fixed $N$ there exists $\beta>0$ such that

$$
\sum_{j=1}^{N} \frac{\epsilon^{2} y_{j}^{2}}{\left(\epsilon+\lambda_{j}\right)^{2}}<\frac{\delta}{2}
$$


for $0<\epsilon<\beta$. Thus

$$
\left\|y_{\epsilon}(T)-\hat{y}\right\|_{L^{2}(\Omega)}^{2}<\delta
$$

for $0<\epsilon<\beta$. The proof is complete.

Definition System (2.1) is said to be approximately controllable if, for any given $\hat{y}$ and $\delta>0$, there exists a control $u(t)$ and a function $y(t)$ such that $(y(t), u(t))$ is a solution of $(2.1)$ and

$$
\|y(T)-\hat{y}\|<\delta
$$

The following theorem is an immediate consequence of Theorem 3.5 .

Theorem 3.6 System (2.1) is approximately controllable.

Remark Approximate controllability is well known for linear parabolic differential equations. Our proof is different in that it is a constructive proof.

\section{A Condition for Exact Controllability}

In this section we give a condition on $\hat{y}$ for the exact controllability of system (2.1). The main tool is the operator $R$ introduced in $\S 2.2$.

Theorem 4.1 Let $R$ be the operator defined in $\S 2.2$ and $\left(\left\{e_{j}\right\}_{j=1}^{\infty},\left\{\lambda_{j}\right\}_{j=1}^{\infty}\right)$ be the eigensystem of $R$. Assume that $R^{-1}$ is the inverse of operator $R$. If

$$
\hat{y}-E(T) y_{0} \in D\left(R^{-1}\right)
$$

then system (2.1) is exactly controllable.

Proof: Assume that

$$
\hat{y}-E(T) v=\sum_{j=1}^{\infty} y_{j} e_{j}
$$

Then

$$
\sum_{j=1}^{\infty} \frac{y_{j}^{2}}{\lambda_{j}^{2}}<\infty
$$

By the proof of Theorem 2.6 we have that

$$
\left\|y_{\epsilon}(T)-\hat{y}\right\|_{L^{2}(\Omega)}^{2}=\sum_{j=1}^{\infty} \frac{\epsilon^{2} y_{j}^{2}}{\left(\epsilon+\lambda_{j}\right)^{2}}
$$


Hence

$$
\begin{aligned}
\left\|y_{\epsilon}(T)-\hat{y}\right\|_{L^{2}(\Omega)} & =\left(\sum_{j=1}^{\infty} \frac{\epsilon^{2} y_{j}^{2}}{\left(\epsilon+\lambda_{j}\right)^{2}}\right)^{\frac{1}{2}} \\
& \leq \epsilon\left(\sum_{j=1}^{\infty} \frac{y_{j}^{2}}{\lambda_{j}^{2}}\right)^{\frac{1}{2}} \leq C \epsilon .
\end{aligned}
$$

Let $\left\{\epsilon_{k}\right\}_{k=1}^{\infty}$ be a sequence such that $\epsilon_{k} \rightarrow 0$ as $k \rightarrow \infty$. From the optimality system (3.1) and the proof of Lemma 2.3 we have that

$$
\begin{aligned}
\left\|y_{\epsilon_{k}}(T)\right\|_{H^{1,2}(Q)} & \leq C\left(\left\|u_{\epsilon_{k}}\right\|_{H^{\frac{1}{4}, \frac{1}{2}(\Sigma)}}+\left\|y_{0}\right\|_{H^{1}(\Omega)}\right) \\
& =C\left(\left\|\frac{p_{\epsilon_{k}}}{\epsilon_{k}}\right\|_{H^{\frac{1}{4}, \frac{1}{2}(\Sigma)}}+\left\|y_{0}\right\|_{H^{1}(\Omega)}\right) \\
& \leq C\left(\left\|\frac{p_{\epsilon_{k}}}{\epsilon_{k}}\right\|_{H^{\frac{1}{2}, 1}(Q)}+\left\|y_{0}\right\|_{H^{1}(\Omega)}\right) \\
& \leq C\left(\left\|\frac{y_{\epsilon_{k}}(T)-\hat{y}}{\epsilon_{k}}\right\|_{L^{2}(\Omega)}+\left\|y_{0}\right\|_{H^{1}(\Omega)}\right) \\
& \leq C_{1}\left\|y_{0}\right\|_{H^{1}(\Omega)}+C_{2} .
\end{aligned}
$$

Thus there exists a subsequence of $\left\{\epsilon_{k}\right\}$, still denoted as $\left\{\epsilon_{k}\right\}$, and $y \in H^{1,2}(Q)$ such that

$$
\lim _{k \rightarrow \infty} y_{\epsilon_{k}}=y \quad \text { weakly in } H^{1,2}(Q)
$$

Using the trace theorem ([1]) and the compact embedding theorem ([1]) in Sobolev spaces we also have that there exists $u \in L^{2}(\Sigma)$ such that

$$
\lim _{k \rightarrow \infty} u_{\epsilon_{k}}=u \text { in } L^{2}(\Sigma)
$$

Also by the trace theorem $([1])$ we have that $y(T)=\hat{y}$ and $y(0)=y_{0}$. We now prove that $(y, u)$ is a solution of (2.1). Since $\left(y_{\epsilon_{k}}(t), u_{\epsilon_{k}}(t)\right)$ is a solution of $(2.2)$

$$
\left(\frac{d y_{\epsilon_{k}}}{d t}, \phi\right)+a\left(y_{\epsilon_{k}}, \phi\right)=<u_{\epsilon_{k}}, \phi>, \quad \text { for } \phi \in H^{1}(\Omega)
$$

Passing to the limit in the above equality we obtain

$$
\left(\frac{d y}{d t}, \phi\right)+a(y, \phi)=<u, \phi>, \quad \text { for } \phi \in H^{1}(\Omega)
$$

Thus $(y, u) \in H^{1,2}(Q) \times L^{2}(\Sigma)$ is a solution of (2.1). This proves that $u$ is an exact control. The proof is complete.

Remark 4.1 From the proof of Theorem 4.1 we see that if (4.1) holds, then

$$
\left\|y_{\epsilon}(T)-\hat{y}\right\|_{L^{2}(\Omega)} \leq C \epsilon
$$


It is easy to show that (4.1) is actually a necessary condition for the above inequality to hold. In fact, assume that the above inequality holds. Then by the proof of Theorem 2.8 we have that

$$
\|y(\epsilon, T)-\hat{y}\|_{L^{2}(\Omega)}^{2}=\sum_{j=1}^{\infty} \frac{\epsilon^{2} y_{j}^{2}}{\left(\epsilon+\lambda_{j}\right)^{2}} \leq C \epsilon^{2} .
$$

Thus

$$
\sum_{j=1}^{\infty} \frac{y_{j}^{2}}{\left(\epsilon+\lambda_{j}\right)^{2}} \leq C
$$

Letting $\epsilon \rightarrow 0$, we obtain (4.1).

Remark 4.2. In [18], Russell gave a condition on exact controllability of system (2.1) using the harmonic analysis method. The condition is

$$
\hat{y}-E(T) v \in e^{C \sqrt{A}}
$$

where $C>0$ is a constant and $A$ is the elliptic operator that appears in (2.1). For $d=1$, this condition implies that $\hat{y}-E(T) v$ is at least a $C^{\infty}$ function. At this moment it is not clear if our condition is weaker. However, if the control is distribution we can show that

$$
D\left(R^{-1}\right)=D(A)=H^{2}(\Omega)
$$

which is much weaker than $D\left(e^{C \sqrt{(} A)}\right)$.

\section{REFERENCES}

1. R. Adams, Sobolev Spaces, Academic, New York, 1975.

2. Yu. V. Egorov, Some problems in the theory of optimal control, Soviet Math. 31962 , 1080-1084.

3. Yu. V. Egorov, Ž. Vyčisl, Mat. i Mat. Fiz. 5 1963, 887-904

4. H. Fattorini, Control in finite time of differential equations in Banach space, Comm. Pure Appl. Math., 19 1996, 7-34.

5. H. Fattorini and D. Russell, Exact controllability theorems for linear parabolic equations in one space dimension, Arch. Rational Mech. Anal. 49 1971, 272-292. 
6. A. Friedman, Partial Differential Equations, Holt, Rinehart, and Winston, New York, 1960.

7. A. Fursikov and O. Imanuvilov, On exact boundary zero controllability of two-dimensional Navier-Stokes equations, Acta Appl. Math. 36 1994, 1-10.

8. A. Fursikov and O. Imanuvilov, On controllability of certain systems stimulating a fluid flow, in: Flow Control, IMA Volumes in Mathematics and Its Applications 68, Springer, New York, 1995, 149-184.

9. L. Gal'chuk, Optimal control of systems described parabolic equations, SIAM. J. Control 7 1969, 546-558.

10. R. Glowinski, C. H. Lee and J. -L. Lions, A numerical approach to the exact boundary controllability of the wave equation (I) Dirichlet controls: Description of the numerical methods, Japan J. Appl. Math. 7 1990, 1-76.

11. M. Gunzburger, L. Hou, and T. Svobodny, Analysis and finite element approximation of optimal control problems for the stationary Navier-Stokes equations with distributed and Neumann controls, Math. Comput. 57 1991, 123-151.

12. J.-L. Lions, Optimal Control of Systems Governed by Partial Differential Equations, Springer, Berlin, 1971.

13. J.-L. Lions, Some Methods in the Mathematical Analysis of Systems and Their Controls, Gordon and Breach, New York, 1981.

14. J.-L. Lions, Some Aspect of the Optimal Control of Distributed Parameter Systems, SIAM, Philadephia, 1972.

15. J.-L. Lions and E. Magnenes, Nonhomogeneous Boundary Value Problems and Applications, Springer, Berlin, 1972.

16. J.-L. Lions, Exact controllability, stabilization and perturbations for distributed systems, SIAM Review 30 1988, 1-68.

17. J. Oden and L. Demkowicz, Applied Functional Analysis, CRC Press, Boca Raton-New York-London-Tokyo, 1996. 
18. D. Russell, A unified boundary controllability theory for hyperbolic and parabolic partial differential equations, Studies in Appl. Math. 52 1973, 189-211.

19. D. Russell, Controllability and stabilizability theory for linear partial differential equations: recent progress and open questions, SIAM Review 20 1978, 639-739.

20. D. Russell, Some remarks on numerical aspects of coefficient identification in elliptic systems, in: Optimal Control of Partial Differential Equations, Birkhauser Verlag- Boston $1984,210-228$.

21. R. Winther, Error estimates for a Galerkin approximation of a parabolic control problem, Ann. Mat. Pura. Appl. 4 1978, 173-206. 